\title{
Carbon abundances of early B-type stars in the solar vicinity $\star, \star \star$
}

\section{Non-LTE line-formation for C II/III/IV and self-consistent atmospheric parameters}

\author{
M. F. Nieva ${ }^{1,2}$ and N. Przybilla ${ }^{1}$ \\ 1 Dr. Remeis Sternwarte Bamberg. Sternwartstr. 7, 96049 Bamberg, Germany \\ e-mail: nieva@sternwarte.uni-erlangen.de \\ 2 Observatório Nacional, Rua General José Cristino 77 CEP 20921-400, Rio de Janeiro, Brazil
}

Received 2 July 2007 / Accepted 18 November 2007

ABSTRACT

\begin{abstract}
Context. Precise determinations of the chemical composition in early B-type stars constitute fundamental observational constraints on stellar and galactochemical evolution. Carbon, in particular, is one of the most abundant metals in the Universe but analyses in early-type stars are known to show inconclusive results. Large discrepancies between analyses of different lines in C II, a failure to establish the $\mathrm{C}$ II/III ionization balance, and the derivation of systematically lower abundances than from other indicators like $\mathrm{H}$ II regions and young FG-type stars all pose long-standing problems.

Aims. We discuss improvements to the non-LTE modelling of the visual line spectrum and to the spectral analysis of early B-type stars, as well as their consequences for stellar parameter and abundance derivations. The most relevant sources of systematic uncertainies and their effects on the analysis are investigated. Consequences for the present-day carbon abundance in the solar vicinity are discussed.

Methods. We present a comprehensive and robust $\mathrm{C}$ II/III/IV model for non-LTE line-formation calculations based on carefully selected atomic data. The model is calibrated with high-S/N spectra of six apparently slow-rotating early B-type dwarfs and giants, which cover a wide parameter range and are randomly distributed in the solar neighbourhood. A self-consistent quantitative spectrum analysis is performed using an extensive iteration scheme to determine stellar atmospheric parameters and to select the appropriate atomic data used for deriving chemical abundances.

Results. We establish the carbon ionization balance for all sample stars based on a unique set of input atomic data. Consistency is achieved for all modelled carbon lines of the sample stars. Highly accurate atmospheric parameters and a homogeneous carbon abundance of $\log (\mathrm{C} / \mathrm{H})+12=8.32 \pm 0.04$ are derived with reduced systematic errors. Present evolution models for massive stars indicate that this value may require only a small adjustment because of the effects of rotational mixing, by $<+0.05$ dex per sample star. This results in a present-day stellar carbon abundance in the solar neighbourhood, which is in good agreement with recent determinations of the solar value and with the gas-phase abundance of the Orion H II region. Our finding of a homogeneous present-day carbon abundance also conforms to predictions of chemical-evolution models for the Galaxy. Moreover, the present approach allows us to constrain the effects of systematic errors on fundamental parameters and abundances. This suggests that most of the difficulties found in previous work may be related to large systematic effects in the atmospheric parameter determination and/or inaccuracies in the atomic data.
\end{abstract}

Key words. line: formation - stars: early type - stars: fundamental parameters - stars: abundances - Galaxy: abundances solar neighbourhood

\section{Introduction}

Studies of the chemical composition of early-type stars provide valuable observational constraints on our understanding of broad fields like stellar evolution, nucleosynthesis, and galactochemical evolution. The accuracy of elemental abundance determinations defines the degree to which theory can be tested. However, quantitative spectroscopy relies itself on many model assumptions. A detailed understanding of the interaction between the radiation field and the plasma in the stellar atmosphere is thus required. Any weakness in the physical model limits the analysis even when high quality in observation and data reduction is achieved.

\footnotetext{
^ Based on observations collected at the European Southern Observatory, Chile, ESO 074.B-0455(A).

$\star \star$ Figures 17 to 22 are only available in electronic form at http://www . aanda.org
}

Concerning Galactochemical evolution (e.g. models of Hou et al. 2000; Chiappini et al. 2001, 2003), early-type stars are important probes for the current chemical composition of the Galaxy. They also provide spatial information of elemental abundances beyond the solar vicinity, allowing Galactic metallicity gradients to be derived (e.g. Gummersbach et al. 1998; Rolleston et al. 2000; Daflon \& Cunha 2004). Abundances from young stars complement data from H II regions, allowing the current status of the nucleosynthesis of metals to be constrained in the course of the cosmic cycle of matter. Pristine abundances can be derived not only for Galactic early-type stars, but also for objects in the metal-poor environments of the Magellanic Clouds, using the present generation of large telescopes and high-resolution spectrographs (e.g. Korn et al. 2002, 2005; Rolleston et al. 2003; Hunter et al. 2007). Such investigations can be extended to more distant galaxies of the Local Group, and even beyond, by analysing intermediate-resolution spectra of early-type supergiants (e.g. Trundle et al. 2002; 
Urbaneja et al. 2005a,b). Moreover, CNO abundances derived from quantitative spectral analyses of massive early-type stars provide fundamental observational constraints for stellar evolution models. Basic stellar parameters and abundance patterns of the light elements resulting from mixing with nuclear-processed matter facilitate an empirical evaluation of different evolution models (e.g. Heger \& Langer 2000; Maeder \& Meynet 2000).

Carbon plays a special rôle among the light elements. It is a primary element created in the fundamental $3 \alpha$-process, and as such, it provides the seed for the subsequent synthesis of all heavier elements (Burbidge et al. 1957; Cameron 1957). Carbon is an essential catalyst for the nucleosynthesis of $\mathrm{H}$ into $\mathrm{He}$ through the CNO cycle in massive and intermediate-mass stars. The element also constitutes the basis of all organic chemistry.

Carbon abundances derived from early-type stars were the subject of numerous spectroscopic studies in the past decades, from the pioneering work of Unsöld (1942) to numerous others since then. The quality of observed data and the complexity of the model calculations and spectral analysis have been improved with great effort over these years, however some inconsistencies remained until the present. The "classical" carbon problem in early-type stars addresses the extremely low abundances derived from the strong C II $\lambda 4267 \AA$ doublet when compared with those derived from weaker lines, as pointed out by Kane et al. (1980), referring to work by Hardorp \& Scholz (1970) and Kodaira \& Scholz (1970). Later on, more complications were found, such as discrepancies from both strong C II $\lambda \lambda 6578 / 82 \AA$ and $4267 \AA$ multiplets, $\lambda \lambda 6578 / 82 \AA$ and the weak lines, among the $C$ II weak lines themselves and in abundances derived from C II and C III.

In nearby, bright, and apparently slow-rotating stars it is possible to derive carbon abundances from weak lines that are less sensitive to the details of the analysis. However, studies of extragalactic or fast-rotating stars are restricted to the stronger lines because of $\mathrm{S} / \mathrm{N}$-constraints or rotational smearing. In those cases, one might be interested to analyse not only the C II $\lambda \lambda 6578 / 82 \AA$ and the $4267 \AA$ multiplets, but also other strong C III or C IV lines, in order to assure ionization equilibrium.

A crucial step in the spectral modelling was to abandon the approximation of local thermodynamic equilibrium (LTE) in line-formation calculations and allow for deviations (nonLTE). Several model atoms have been discussed in the literature (Lennon 1983; Sakhibullin 1987; Eber \& Butler 1988; Grigsby et al. 1992; Sigut 1996; Przybilla et al. 2001; Lanz \& Hubeny 2003, 2007). The model atom of Eber \& Butler found wide application to abundance analyses of mostly unevolved early-type stars in the solar neighbourhood (e.g. Gies \& Lambert 1992; Kilian 1992; Cunha \& Lambert 1994; Gummersbach et al. 1998; Andrievsky et al. 1999; Daflon et al. 1999, 2001a,b).

The C II $\lambda 4267 \AA$ multiplet poses a challenge to non-LTE line-formation calculations (e.g. Lambert 1993; Sigut 1996). Even in non-LTE studies, this and the $\lambda \lambda 6578 / 82 \AA$ multiplet failed to reproduce observation consistently (Grigsby et al. 1992; Hunter et al. 2007). Systematically lower abundances are derived than from other weaker C II lines (Gies \& Lambert 1992), as in the LTE case. Observed trends for C III lines may also be poorly matched by non-LTE model calculations (Grigsby et al. 1992). A failure to establish the C II/III ionization equilibrium in nonLTE has also been reported. Differences in abundance from the two ions can amount to a factor 5-10 (Daflon et al. 2001b; Hunter et al. 2007). There are even more inconsistencies with published carbon abundances from early-type stars in a broader context that require other explanations, as most of the published studies avoid the lines sensitive to non-LTE effects.

A comparison of the available studies of early B-type stars indicates that present-day carbon abundances in the solar vicinity are highly inhomogeneous (even for stars within a single cluster) and largely sub-solar. This contrasts the findings of a uniform abundance in the gas-phase of the interstellar medium (ISM) within $1.5 \mathrm{kpc}$ of the Sun (Sofia \& Meyer 2001, and references therein). It cannot be understood from current stellar and Galactochemical evolution models either. Young massive stars that form out of a molecular cloud on a short timescale can be expected to show a homogeneous carbon abundance ${ }^{1}$, which also coincides with that of their surrounding H II nebula. Moreover, this abundance is expected to be higher than for objects from previous generations of star formation in their neighbourhood, like young ( $\leq 2 \mathrm{Gyr}$ ) F and G stars and the Sun. In reality, significant systematic differences exist (see e.g. Sofia \& Meyer 2001; Herrero 2003), casting doubt on the reliability of carbon abundances derived from early B-type stars.

Quantitative spectroscopic studies depend on the quality of the observational data, the physical approximations made in the modelling approach (stellar atmospheres, model atoms), and the analysis technique. The analysis procedure also involves several steps (data reduction, stellar parameter determination, and abundance derivation), which are all critical for the quality of the final results. It is difficult to control systematic effects in the whole process. Therefore, the sources of discrepant results from the literature are hard to be identified.

One underestimated problem in deriving the chemical composition is certainly the selection of the input atomic data for non-LTE line-formation calculations (see e.g. Sigut 1996, for $\mathrm{C}$ II). Even when the model atmosphere computations reproduce realistically the plasma conditions in the stellar photosphere, use of different atomic data for the line-formation calculations can result in large systematic errors in the final abundances. The structure of atoms and therefore the atomic line spectra are governed by the laws of quantum mechanics; i.e., they are essentially independent of the environment the atoms are located in. This property can be used to calibrate model atoms for non-LTE calculations, by demanding that the model should reproduce observations reliably over the entire parameter space of relevance. In practice, this means that model atoms need to be tested for a few cases of well-established plasma conditions. The atmospheres of early B-type stars far from the Eddington limit provide such testbeds, as they can be described well by rather simple physical models. A small sample of stars, spanning the relevant range in spectral type and luminosity, is sufficient for such tests.

The present study aims to solve several inconsistencies concerning the derivation of carbon abundances in early B-type stars and to apply the new modelling and analysis technique to stars of the solar vicinity. It focuses on both i) the critical selection of appropriate atomic input data from ab-initio calculations and approximation formulae and ii) a self-consistent derivation of the atmospheric parameters of the sample stars from ionization equilibria. This detailed and reliable study allows us to derive abundances with unprecedented accuracy for a so far small but very well constrained sample of stars covering strategic points of the stellar parameter space and randomly distributed in OB associations and in the field of the solar neighbourhood.

${ }^{1}$ Carbon experiences a slight depletion in the course of the $\mathrm{CNO}$ cycle, which may become observable at the stellar surface because of rotational mixing, see Sect. 6 for a discussion. 
This study is part of our efforts to improve on the accuracy of quantitative spectroscopy in early B-type stars (Nieva \& Przybilla 2007: Paper I; Nieva \& Przybilla 2006: Paper II) and related objects (Przybilla et al. 2005, 2006a-c). The hybrid nonLTE approach (LTE model atmospheres + non-LTE line formation) was shown to be adequate for analyses of OB-type main sequence and giant stars in Paper I, allowing highly consistent results to be derived. In particular, practically all measurable $\mathrm{H}$ and $\mathrm{He}$ lines from the Balmer jump to the near-IR (where available) were reproduced with a single model for each of the present sample stars, meeting a crucial boundary condition for all further analyses. Paper II discusses a solution to the "classical" carbon problem, i.e. discrepant carbon abundances from C II $\lambda 4267 \AA$ and weaker lines, and also a solution to the notorious discrepancy of the prominent $\lambda \lambda 6578 / 82 \AA$ and the $4267 \AA$ multiplets. There, consistency was achieved for up to $21 \mathrm{C}$ II features of our sample stars. Those results are part of the present work, which discusses the topic in a much broader context. Here, we present for the first time a quantitative agreement of abundances from $\mathrm{C}$ II and C III lines for this kind of star. We also study C IV lines and establish the C II/III/IV ionization equilibrium in a self-consistent way, never considered before in similar work. The linelist reaches 40 features. In the present work we investigate the systematic errors arising from uncertainties in the atomic data and stellar parameters and quantify the difference between LTE and non-LTE analyses, for the first time in this extent. The implications of effective temperature scales for abundance determitations are discussed. This extensive study provides us with reliable carbon abundances for a discussion in the context of galactochemical evolution.

The paper is organised as follows. Section 2 describes the model calculations including a description of the carbon model. Section 3 describes the model calibration via an extensive and self-consistent iteration and the sensitivity of carbon line-formation calculations to atomic data and atmospheric parameter variations. Section 4 summarises the final results for parameters and carbon abundances of individual lines for the sample stars. In Sect. 5 we provide a comparison of our results to previous studies and the conclusions on the present-day carbon abundance in the solar neighbourhood are discussed in Sect. 6. A summary is given in Sect. 7.

\section{Model calculations}

The non-LTE line-formation computations for carbon follow the methodology discussed in our previous study of the hydrogen and helium spectra in early-type stars (Paper I). A hybrid nonLTE approach is employed to solve the restricted non-LTE problem on the basis of prescribed LTE atmospheres. This technique provides an efficient way to compute realistic synthetic spectra in all cases where the atmospheric structure is close to LTE. The computational efforts can thus be concentrated on robust nonLTE line-formation calculations.

\subsection{Model atmospheres and programs}

For the spectral synthesis, the model atmospheres are computed with the ATLAS9 code (Kurucz 1993b), which assumes plane-parallel geometry, chemical homogeneity, as well as hydrostatic, radiative and local thermodynamic equilibrium (LTE). Line blanketing is realised via consideration of opacity distribution functions (ODFs, Kurucz 1993a). We adopt here standard solar abundances (Grevesse \& Sauval 1998) in all computations, as in Paper I. Helium abundance and microturbulence are adjusted according to the results from the quantitative analysis of the programme stars - requiring some iteration, as explained below. The model atmospheres are held fixed in the non-LTE calculations.

Non-LTE level populations and model spectra are obtained with recent versions of DETAIL and SURFACE (Giddings 1981; Butler \& Giddings 1985; both updated by Butler). The coupled radiative transfer and statistical equilibrium equations are solved with DETAIL, employing an accelerated lambda iteration scheme of Rybicki \& Hummer (1991). This allows even complex ions to be treated in a realistic way. Continuous opacities due to hydrogen and helium (for actual abundances) are considered in non-LTE, and line blocking is accounted for in LTE via Kurucz' ODFs. Synthetic spectra are calculated with SURFACE, using refined line-broadening theories. Microturbulence is consistently accounted for in both steps with DETAIL and SURFACE.

Non-LTE level populations for hydrogen and He I/II are computed using recent model atoms by Przybilla \& Butler (2004) and Przybilla (2005), respectively. The synthetic $\mathrm{H}$ and $\mathrm{He} / \mathrm{II}$ lines give excellent agreement with observations, in the optical and in the near-IR (Paper I). This is a prerequisite for modelling metal lines that overlap with the (broad) hydrogen or helium features. In particular, the C II $\lambda \lambda 6578 / 82 \AA$ doublet is affected in the present case.

\subsection{The C II-IV model atom}

We give a short summary of the input atomic data for constructing the $\mathrm{C} \mathrm{II/III/IV} \mathrm{model} \mathrm{atom} \mathrm{in} \mathrm{this} \mathrm{section.} \mathrm{A} \mathrm{more} \mathrm{detailed}$ description of our motivation for choosing these atomic data will be given in Sect. 3.2, as this turned out to be critical for realistic modelling of the observed spectra.

C II. This model ion considers $L S$-coupled terms up to principal quantum number $n=10$ and angular momentum $\ell=9$ (66 levels) explicitly in the non-LTE calculations, with all finestructure sub-levels combined into one. Additional levels up to $n=14$ are computed in LTE relative to the ground state of C III. Level energies are adopted from Moore (1993), Sigut (1996), and Quinet (1998). The doublet and quartet spin systems are treated simultaneously. Oscillator strengths ( $g f$-values) from three sources are considered: fine-structure data from abinitio computations using the multiconfiguration Hartree-Fock method in the Breit-Pauli approximation of Froese Fischer \& Tachiev (2004, FFT04), data from an application of the BreitPauli $R$-matrix method (Nahar 2002a, N02a), and results obtained in the Opacity Project (OP) from the $R$-matrix method assuming LS-coupling (Yan et al. 1987). Our primary source of $g f$-values is FFT04, followed by OP and N02a for the remaining transitions. Intercombination transitions are neglected because of very small oscillator strengths. Photoionizations crosssections are adopted from the OP (Yan et al. 1987) for levels up to $n=9$ and $\ell=3$ with a correction of the threshold frequencies to observed values and from Nahar \& Pradhan (1997: NP97) for the remainder. Effective collision strengths for electron impact excitation among the lowest 16 LS-states are adopted from $\boldsymbol{R}$-matrix computations of Wilson et al. (2005, 2007). An empirical increase by a factor two was applied to the $3 \mathrm{~s}^{2} \mathrm{~S}-3 \mathrm{p}^{2} \mathrm{P}^{\mathrm{o}}$ data (see Sect. 3.2). Collisional excitation for transitions without detailed data are treated using the Van Regemorter (1962) approximation - in the optically allowed case - and via the semiempirical Allen (1973) formula in the optically forbidden case. Collision strengths $\Omega$ varying between $0.01(\Delta n \geq 4)$ to 100 
$(\Delta n=0)$ are employed, as suggested by evaluating the detailed data from ab-initio computations of Wilson et al. (2005, 2007). Collisional ionization rates are evaluated according to the Seaton (1962) approximation. Threshold photoionization crosssections are adopted from OP and NP97, allowing for an empirical correction of one order of magnitude higher for the $6 \mathrm{f}^{2} \mathrm{~F}^{\circ}$ and $6 \mathrm{~g}^{2} \mathrm{G}$ levels - corresponding to the upper levels of the $\mathrm{C}$ II $\lambda \lambda 6151$ and $6462 \AA$ transitions, respectively.

C III. This model accounts for $L S$-terms up to $n=7$ and $\ell=7$ (70 levels) explicitly in the statistical equilibrium calculations. In a similar way to $\mathrm{C}$ II, levels up to $n=14$ are computed in LTE relative to the ground state of the next ionization stage. The two spin systems (singlet and triplet) are treated simultaneously. Level energies are taken from the NIST database ${ }^{2}$ and energies for 9 of the highest levels are adopted from N02a. Two sources are considered for oscillator strengths: N02a and additional values from Eber (1987). Intercombination transitions are also implemented when their values are non-negligible $\left(f>10^{-4}\right)$. Photoionization cross-sections are taken from S. Nahar's webpage $^{3}$. Maxwellian-averaged collision strengths for electronimpact excitation among the lowest 24 terms are adopted from the $R$-matrix computations of Mitnik et al. (2003). Collisional excitation for the remaining transitions and collisional ionization are treated in analogy to the $\mathrm{C}$ II ion, using appropriate $g f$-values and threshold photoionization cross-sections.

C IV. $L S$-terms up to $n=10$ and $\ell=9$ (53 levels) are treated explicitly. Additional levels up to $n=14$ are computed in LTE relative to the ground state of $\mathrm{C} \mathrm{V}$. Oscillator strengths from $a b$-initio calculations using the Breit-Pauli $\boldsymbol{R}$-matrix method (Nahar 2002b) are adopted. Photoionization cross-sections are also taken from Nahar's webpage. Effective collision strengths for electron impact excitation of transitions among the lowest 24 fine-structure levels are taken from Aggarwal \& Keenan (2004) and subsequently co-added. All remaining transitions, along with collisional ionization, are treated in analogy to C II.

The resulting $\mathrm{C}$ II/III/IV model atom accounts for more than 1300 radiative and more than 5300 collisional transitions and $\sim 200 L S$-coupled energy levels. Over 20000 frequency points allow the detailed resonance structure of the photoionization cross-sections to be sampled with $<0.5 \AA$ resolution. Accuracies of the atomic data can range from a typical $10-20 \%$ for ab-initio computations to orders of magnitude for approximation formulae. Finally, Voigt profiles are adopted in the formal solution using SURFACE. Wavelengths and oscillator strengths of most of the observed transitions are taken from Wiese et al. (1996). For C II $\lambda \lambda 6151.3 / 5$ and $6461.9 \AA$, the wavelengths are adopted from Kurucz \& Bell (1995) and the $f$-values from N02a. Radiative damping parameters are calculated from OP lifetimes and coefficients for collisional broadening by electron impact are adopted from Griem (1974, for the C II $\lambda 4267 \AA$ doublet) or computed according to Cowley (1971). Detailed tabulations from quantummechanical computations for Stark broadening of several C IV transitions (Schöning 1993) are also used. The spectral lines used for abundance analysis are listed later in Table 3.

\footnotetext{
2 http://physics.nist.gov/PhysRefData/ASD/indices.html ${ }^{3}$ http://www-astronomy. mps. ohio-state. edu/ nahar/px. html
}

\section{Simultaneous C II/II//V model-atom calibration and atmospheric parameter determination}

A reliable set of atomic data considered in model atoms for non-LTE calculations can be selected by demanding that the model reproduce the observations over the entire stellar parameter space of relevance. This procedure can be regarded as a "calibration" and requires precise atmospheric parameters for the test sample stars. These should be free of systematic errors to prevent one from being misled when optimising the selection of input atomic data. Unfortunately, the parameters of stars are a priori not known, they also need to be inferred from observations. Consequently, a simultaneous solution for an optimal set of input atomic data and of all stellar parameters is required. These parameters should also allow the hydrogen and helium spectra to be reproduced.

A sample of six apparently slow-rotating B-type dwarfs and giants (spectral class B0-B2, luminosity class V-III) randomly distributed in Galactic OB associations and in the field of the solar vicinity are taken as calibration stars. The observational data consists of high-resolution spectra with broad wavelength coverage at very high- $\mathrm{S} / \mathrm{N}$ (up to $\sim 800$ in the Johnson $B$-band), obtained with FEROS on the ESO $2.2 \mathrm{~m}$ telescope (La Silla, Chile); see Paper I for details.

\subsection{Extensive iteration on stellar and atomic variables}

The atmospheric parameter derivation and selection of input atomic data are simultaneously performed in an extensive iteration process. When possible, we try to separate the effects of the fundamental parameters and the atomic data on the synthetic spectra - the basis for the comparison with observation - in order to achieve a better understanding of the problem. This is facilitated by boundary conditions, like the ionization balance (all ionization stages of an element are required to indicate the same abundance) or the rules and regularities of atomic physics.

The iteration is performed on effective temperature $T_{\text {eff }}$ and surface gravity $\log g$, as well as on micro-, macroturbulent and projected rotational velocities ( $\xi, \zeta$ and $v \sin i$, respectively), helium and carbon abundances (hereafter $\varepsilon(\mathrm{He})^{4}$ and $\varepsilon(\mathrm{C})$, respectively), and on different sets of atomic data. Only the metallicity is fixed to a standard solar value (Grevesse \& Sauval 1998), a not too critical assumption that is furthermore validated a posteriori (Przybilla et al. 2008, in prep.). This comprises an enormous number of variables (atmospheric parameters and atomic data) in the iterative scheme summarised as a flux diagram in Fig. 1. The first step concerning the $\mathrm{H} / \mathrm{He}$ spectrum is solved in Paper I. There, the $\mathrm{He} / \mathrm{II}$ ionization equilibrium is the main indicator for $T_{\text {eff }}$ (for the hotter stars), all Balmer lines for $\log g$, the He II lines for $\xi$, and all He lines for $\zeta$ and $v \sin i$. The second step, involving carbon, is required for a fine tuning of the atmospheric parameter determination since the metal lines are more sensitive to parameter variations than the $\mathrm{H}$ and $\mathrm{He}$ lines. Therefore it is possible to derive them with better precision than only from hydrogen and helium but, at the same time, consistently within the error limits. Effective temperature and $\log g$ are refined by establishing the $\mathrm{C} \mathrm{II/III/IV} \mathrm{ionization} \mathrm{equilibrium} \mathrm{in} \mathrm{the} \mathrm{hottest}$ stars and the $\mathrm{C}$ II/III ionization balance in the cooler stars. The microturbulent velocity is inferred in the standard way by demanding the carbon abundances of the individual lines to be independent of equivalent width. Macroturbulent and projected rotational velocities are determined by detailed fitting of the

${ }^{4}$ Using the standard logarithmic scale $\varepsilon(\mathrm{X})=\log (\mathrm{X} / \mathrm{H})+12$. 
Step 2

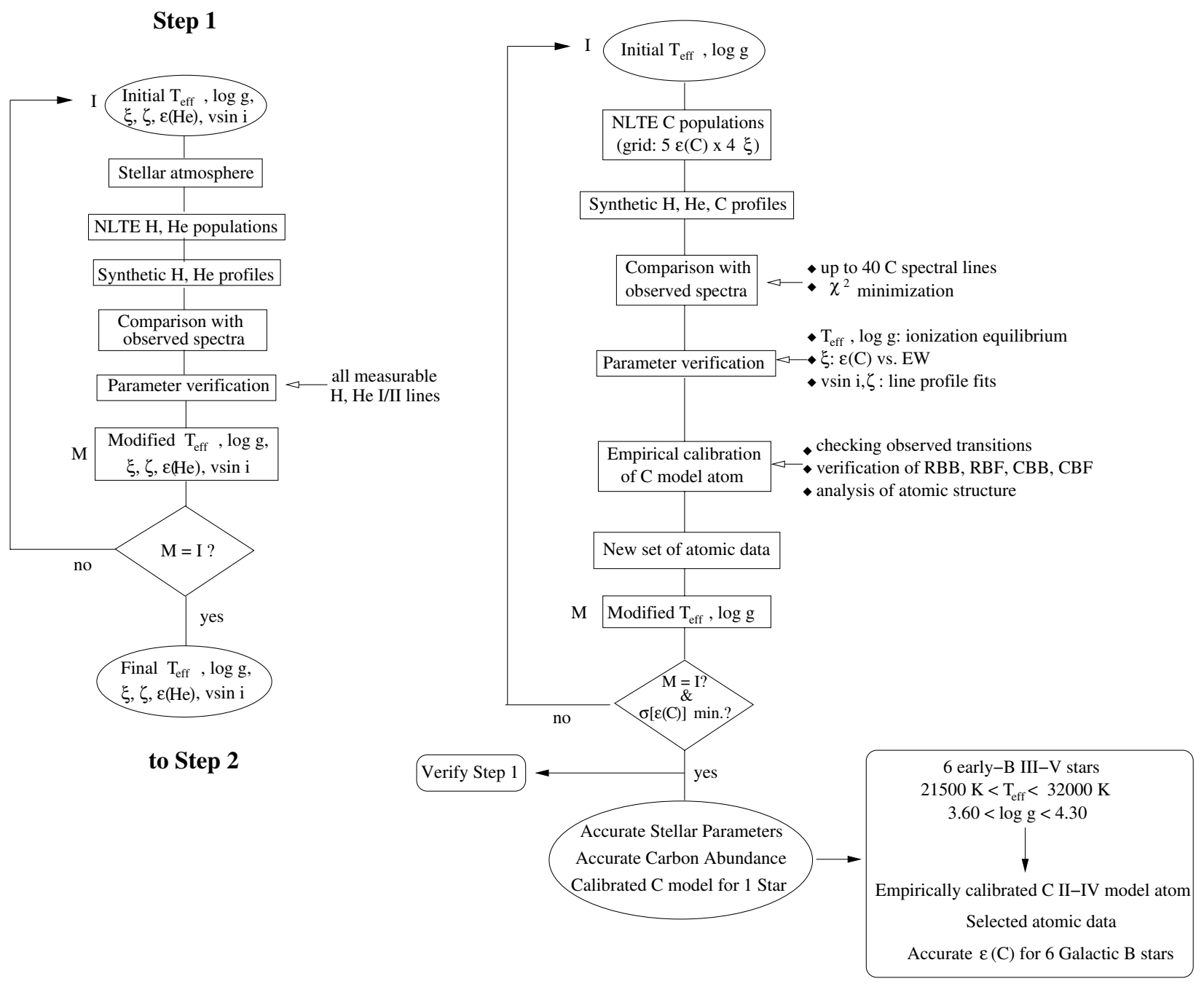

Fig. 1. Flux diagram of our extensive iterative procedure for the carbon model-atom calibration and the simultaneous precise determination of stellar parameters, as applied to each programme star. RBB/CBB: radiative/collisional bound-bound, RBF/CBF: radiative/collisional bound-free transition data, EW: equivalent width. Step 1 is discussed in Paper I, the sensitivity of C lines to different sets of atomic data in Sect. 3.2 and the sensitivity of $\mathrm{C}$ to parameter variations in Sect. 3.4 of the present paper. See the text for details.

carbon line profiles. Line fits are performed on the basis of small grids of synthetic spectra with different $\xi$ and $\varepsilon(\mathrm{C})$ via $\chi^{2}$-minimization.

Since different sets of atomic data were available, we had, first of all, to compare them and judge their reliability to minimize the uncertainties in the $\varepsilon(\mathrm{C})$-determination. Starting from an initial model atom we used our expertise for stepwise improvement, guided by a careful analysis of the atomic structure of the model ions and the reactions of the spectrum synthesis to parameter variations. Over 100 models were built until consistency with all sample stars was achieved simultaneously. Some examples of the effects from different model atom realisations on the spectrum synthesis are given in the next section.

The atmospheric parameters derived from $\mathrm{C}$ ionization equilibrium in Step 2 are verified by re-iterating Step 1 as a final check for consistency. Excellent agreement with the observed $\mathrm{H}$ and $\mathrm{He}$ spectra is obtained in all cases, simultaneously in the visual and the near-IR (where available). In addition, consistency of the observed and model spectral energy distributions (Paper II) is found for the final set of stellar parameters.
By applying this procedure to all programme stars, it was possible to calibrate the $\mathrm{C}$ II-IV model over the entire parameter range $\left(21500 \leq T_{\text {eff }} \leq 32000 \mathrm{~K}, 3.60 \leq \log g \leq 4.30\right.$, for dwarfs and giants), resulting in a final reference set of atomic data. Note that the high-quality spectra available to us are essential for this success. They allowed us to analyse a wide variety of $\mathrm{C}$ lines, many of which have never been considered before in the study of early B stars. Some weak lines turned out to be highly sensitive to non-LTE effects and/or to atmospheric parameter variations, namely the C IV lines and C II $\lambda \lambda 6151$ and $6462 \AA$, which change from absorption to emission at higher temperatures (discussed in Paper II). The reproduction of the observed trends, despite this high sensitivity, puts strong constraints on the robustness of the final model atom. Note also that the presence of lines from three C ionization stages, in the hotter stars of our sample, allows $T_{\text {eff }}$ and $\log g$ to be derived from the ionization equilibrium alone, independent of other indicators. The strength of this calibration lies in the simultaneous analysis of the large number of $\mathrm{C}$ lines of different ionization stages in stars covering a wide parameter range. In this way we were able to constrain a final set of atomic data independent of any specific stellar atmosphere environment. 


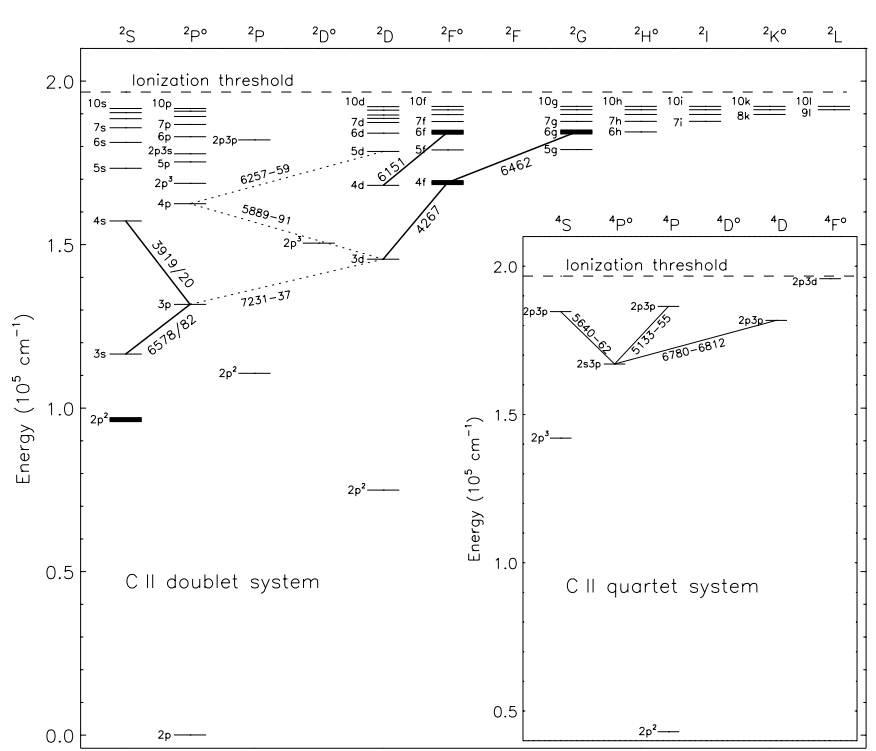

Fig. 2. Grotrian diagram for the $\mathrm{C}$ II doublet and quartet spin systems. The observed multiplets in our spectra analysed here are identified. Levels: those marked in bold correspond to levels discussed in Figs. 3 and 6. Multiplet transitions: those marked by thick lines are highly sensitive to variations of input data for photoionization and collisional cross-sections and for collisional excitation (discussed in Figs. 4, 6 and 7, respectively). The latter and those marked by thin lines are considered in our linelist (Table 3) for abundance derivation. Those marked by dotted lines are excluded from the analysis because of contamination with telluric lines $(\lambda \lambda 6257-59,7231-37 \AA)$ or they are too weak even at low $T_{\text {eff }}(\lambda 5889-91 \AA)$. Nevertheless they are accounted for in the calculations of the level populations.

This reference model can be used in further applications with a simplified iterative scheme, where the only remaining variables will be the atmospheric parameters and the $\mathrm{C}$ abundance.

\subsection{Sensitivity of carbon lines to atomic data}

Reliable level populations are a prerequisite for any accurate non-LTE analysis. They can be obtained only when I) the local temperatures and particle densities are known (i.e. the atmospheric structure), II) the radiation field is realistic, III) all relevant processes in the statistical equilibrium equations are taken into account, and IV) high-quality atomic data are available. In particular, I) and II) require a realistic physical model of the stellar atmosphere (see Paper I for a discussion of the hybrid nonLTE approach) and accurate atmospheric parameter determination. Points III) and IV) are related to the model atoms for the non-LTE calculations. Shortcomings in any of I)-IV) result in increased uncertainties/errors of the analysis.

Due to the interdependency of all transitions (over 6000 in this case) and the non-local character of the radiation field, even a restricted non-LTE problem like the one investigated here is highly complex. It is impossible to quantify a priori the sensitivity of the spectral lines to variations in some of the atomic input data. Therefore, one of the few remaining reasons for the wide spread of carbon abundances found in the literature (see Sect. 5) may be different realisations of model atoms (levels/transitions considered, atomic data, approximations). Choosing an optimum set of input atomic data is not trivial and the construction of reliable model atoms for non-LTE calculations requires a calibration, guided by extensive comparisons with observation. In the following we summarise the experiences made in this process.
The comparison of observation with model spectra for C III and CIV, as computed with our initial model ions, reveals little need for improvement in the studied stellar parameter range. Both ions are relatively simple, showing (earth)alkali electron configurations, which hardly challenge the ab-initio computations. However, the actual choice of radiative and collisional data turned out to be a critical factor for the line-formation computations of non-LTE-sensitive transitions in C II, which is known to be problematic from the literature (e.g. Lambert 1993; Sigut 1996). This was briefly summarised in Paper II but not discussed in detail. Here we provide a few comparisons of atomic data available from the literature. The influence of atomic data on selected C II lines is also addressed. These transitions are highlighted in the Grotrian diagram of our C II model (Fig. 2), which will help us to illustrate some of the channels leading to the marked non-LTE sensitivity.

Photoionization cross-sections. The strength of a spectral line can be strongly influenced by photoionizations that may impact level populations decisively. On the other hand, photoionization rates $R_{i j}$ depend implicitly on the level populations. The largest contribution to the integral $R_{i j}$ comes from frequencies with large flux and large cross-section. The flux maximum in early B stars is located longward of the Lyman jump. The ionization of C II is essentially determined by the rates from the highly-populated ground-state and the low-excitation levels. The relative importance of the low-excitation levels may be strengthened in cases where the ground state ionization potential coincides with that of a major opacity contributor, $\mathrm{He} \mathrm{I}$ in the present case. Photoionizations from the ground state may then be less efficient because of the reduced stellar flux shortward of the ionization edge. Recombinations, on the other hand, are important for the population of high-excitation levels (preferentially at high $\ell$, i.e. states with large statistical weight), which then couple to the low-lying states via recombination cascades. For the case of C II, this implies: a) an increased sensitivity of the C II/III ionization balance to the exact run of the photoionization crosssections of levels at low excitation energies and b) an increased sensitivity of transitions like C II $\lambda \lambda 6151,6462$, and $4267 \AA$ to non-LTE effects because of their participation in the recombination cascade.

A comparison of total photoionization cross-sections from OP (Yan \& Seaton 1987) and NP97 for two levels (marked in the Grotrian diagram, Fig. 2) is given in Fig. 3. The first term, $2 \mathrm{p}^{2}{ }^{2} \mathrm{~S}$, is not directly involved in the formation of the C II $\lambda \lambda 4267$, 6151 and $6462 \AA$ transitions. However, it is populated considerably and therefore contributes to the $\mathrm{C} \mathrm{II} / \mathrm{III}$ ionization balance. The cross-section for photoionization from this level to the ground state of $\mathrm{C}$ III is small because of radiative selection rules. Shortward of $\sim 700 \AA$ the cross-section rises by about two orders of magnitude as the optically allowed channel of photoionization to the first excited level of C III opens. The overestimation of the photoionization rate from the $2 \mathrm{p}^{2}{ }^{2} \mathrm{~S}$ state by adopting the total instead of partial cross-sections is however insignificant. The contribution of wavelengths below $\sim 700 \AA$ to $R_{i j}$ is e.g. less than $1 \%$ in the example discussed below. Note the wavelength shifts in the resonance structures of the OP and the NP97 data. This results in different contributions of the region between the threshold and the Lyman edge to the integral in $R_{i j}$, which affects the photoionization rates considerably. On the other hand, the photoionization cross-sections for $4 \mathrm{f}^{2} \mathrm{~F}^{0}$ agree well, except for the resonance structure at shortest wavelengths where the stellar flux becomes negligible. Consequently, both data (and many others for highly-excited levels) are exchangeable without 

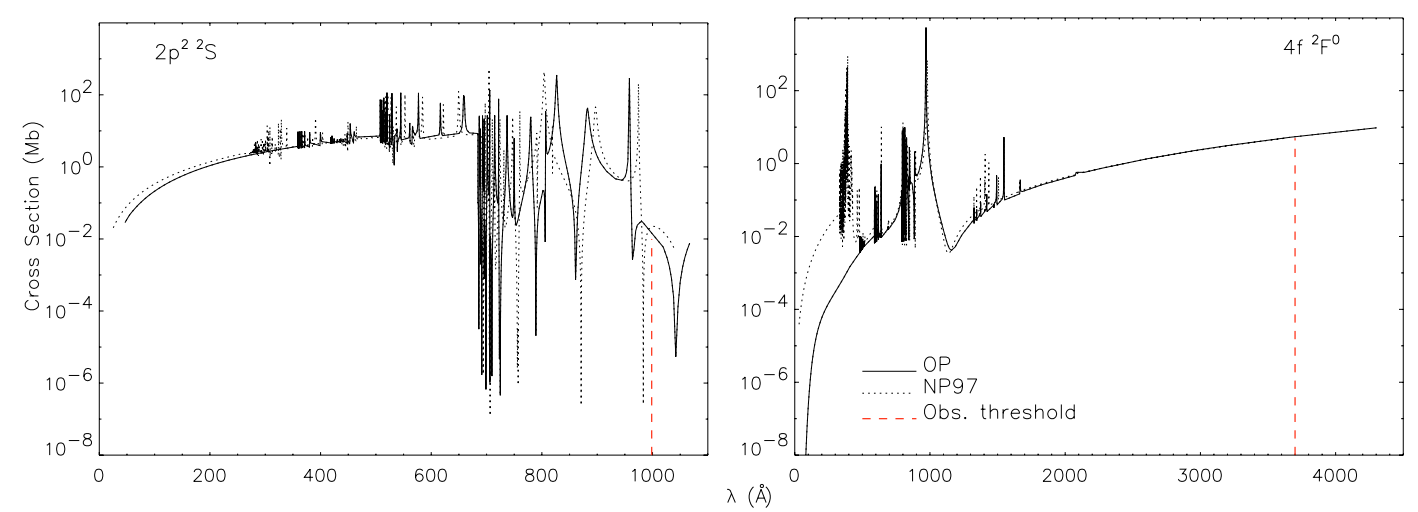

Fig. 3. Comparison of C II photoionization cross-sections from the Opacity Project (Yan \& Seaton 1987) and Nahar (1997) for $2 \mathrm{p}^{2}{ }^{2} \mathrm{~S}$ and $4 \mathrm{f}^{2} \mathrm{~F}^{\mathrm{o}}$ as a function of wavelength. See the text for a discussion.

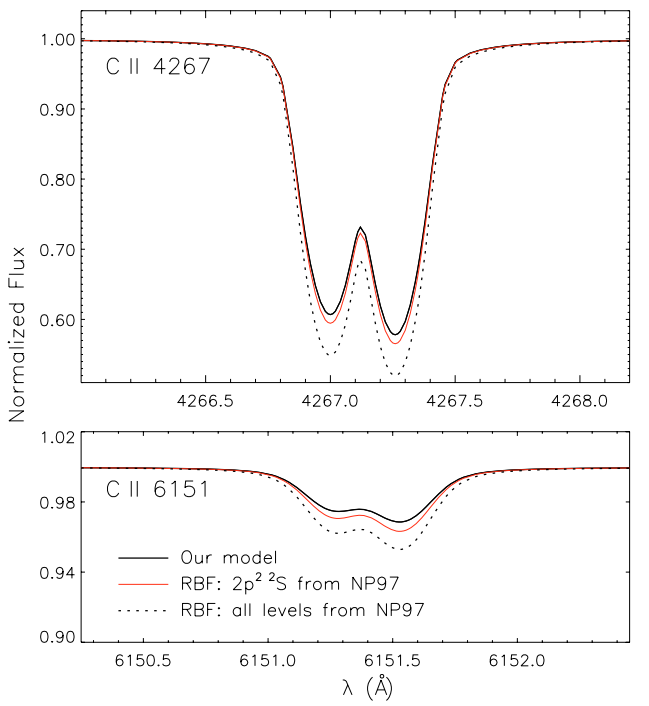

Fig. 4. Sensitivity of line profiles of two C II transitions to variations in photoionization cross-sections. The calculations are made for three different model atoms using the same model atmosphere (as appropriate for HR 5285): our reference model atom, the reference model atom with cross-sections for $2 \mathrm{p}^{2}{ }^{2} \mathrm{~S}$ from NP97, and the reference model atom with all photoionization data replaced by values from NP97. The profiles are not convolved for effects of rotation or instrumental broadening.

showing consequences for the spectrum synthesis computations. We adopt experimental threshold wavelengths.

On the other hand, for $2 \mathrm{p}^{2}{ }^{2} \mathrm{~S}$ and other levels at lower excitation energy, there is a non-negligible effect on the $\lambda \lambda 4267$ and $6151 \AA$ transitions when exchanging both data, as can be seen in Fig. 4. In an extreme case, accounting for photoionization cross-sections from NP97 for all levels results in a very strong C II $\lambda \lambda 4267 \AA$ line. A reduction of the $\mathrm{C}$ abundance by up to $\sim 0.8$ dex is required to fit the observed line profile in our calibration stars with such a model atom. As a consequence of this we give preference to the OP data over the cross-sections of NP97 in our final model atom ("model of reference"), which helps us to reproduce observation over the entire parameter range consistently.

Oscillator strengths. Comparisons between multiplet $f$-values from three sources of ab-initio computations are shown in Fig. 5: data based on I) the multiconfiguration Hartree-Fock method in the Breit-Pauli approximation of FFT04, II) the BreitPauli $R$-matrix method of $\mathrm{N} 02$ a and III) results from $R$-matrix calculations assuming LS-coupling, as obtained by the Opacity

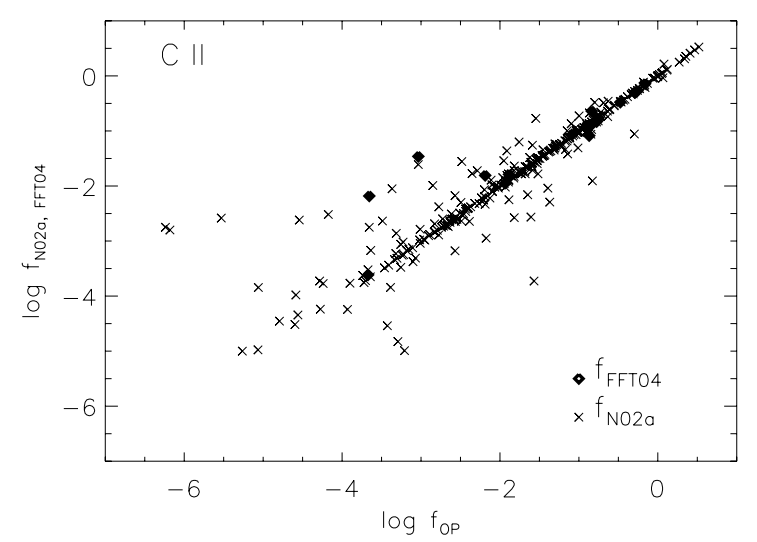

Fig. 5. Comparison of oscillator strengths from Nahar (2002a: N02a) and Froese Fischer \& Tachiev (2004: FFT04) vs. values from the Opacity Project (Yan et al. 1987: OP).

Project (Yan et al. 1987). The primary source of $f$-values is FFT04, which should be the most accurate. Our preference for the OP over the N02a data is motivated by the good agreement of the former with FFT04 (with two exceptions), while oscillator strengths from N02a may show large differences for several lines. Data from N02a is therefore adopted only in the cases where the other sources do not provide information.

Collisional ionization cross-sections. Details of the collisional ionization cross-sections are important mostly for highexcitation levels $(n>5)$. Only for these are a significant amount of electrons in the Maxwell distribution energetic enough to overcome the threshold for the reaction. We used the approximation of Seaton (1962), which is expected to be accurate to an order of magnitude at best, to evaluate the collisional ionization rates because of a lack of any data from ab-initio computations. Good agreement between the modelling and most observed $\mathrm{C}$ II lines is achieved (see below) when using the standard recipe of applying threshold photoionization cross-sections, except for two observed transitions. Improvements for the C II $\lambda \lambda 6151$ and $6462 \AA$ lines may be obtained by an empirical scaling of threshold cross-sections for the $6 f^{2} \mathrm{~F}^{\circ}$ and $6 \mathrm{~g}{ }^{2} \mathrm{G}$ levels, the upper levels of the transitions. An increase by a factor 10, i.e. within the expected uncertainties, helps to reproduce observation over the entire atmospheric parameter range. The effects of scaled collision rates for those line profiles are shown in Fig. 6, as well as C II $\lambda 4267 \AA$, which remains practically unaffected. Larger scaling factors are empirically excluded. 

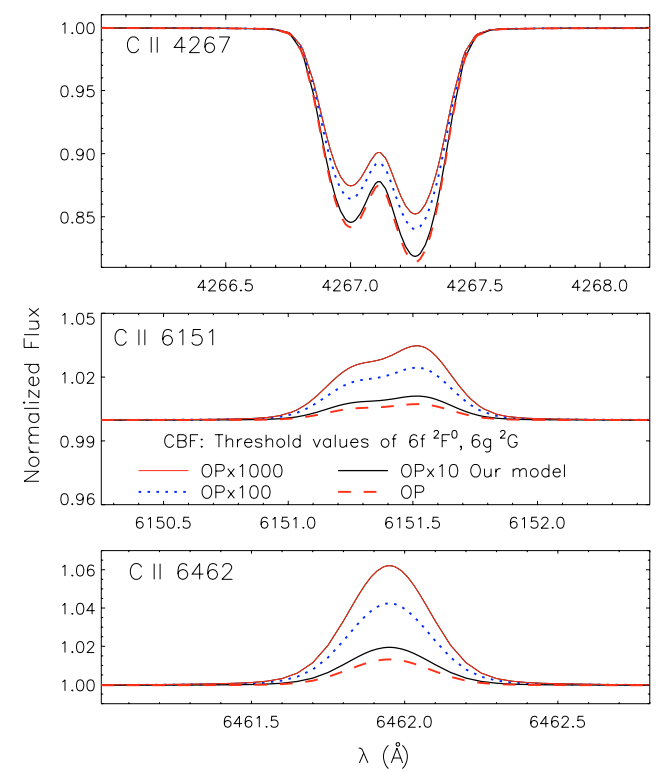

Fig. 6. Reactions of three highly non-LTE-sensitive lines to changes in collisional ionization cross-sections. The modifications are made for two energy levels directly involved in the formation of C II $\lambda \lambda 6151$ and $6462 \AA$ and indirectly in C II $\lambda 4267 \AA$. The calculations are made for different model atoms with specific values of the reaction cross-section at threshold and the same set of atmospheric parameters (for $\tau$ Sco).

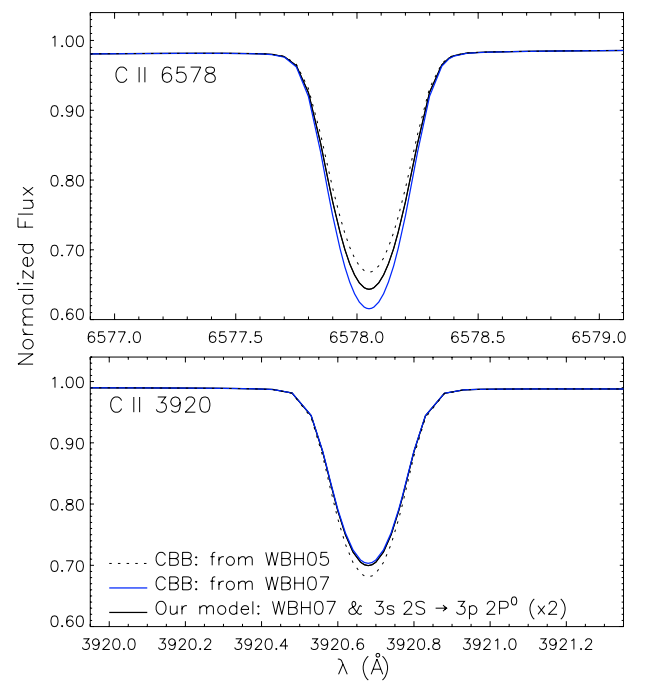

Fig. 7. Effect of employing different effective collision strengths on the C II $\lambda \lambda 6578$ and $3920 \AA$ lines (for HR 1861). Only variations of the data from Wilson et al. $(2005,2007)$ are considered.

Collisional excitation cross-sections. The collisional data used in a model atom will also have an influence on the predicted line profiles. Accurate data from $a b$-initio calculations for larger sets of transitions have only recently become available. We employed effective collision strengths of Wilson et al. (2005) to construct the $\mathrm{C}$ II model. A later revision of part of the data (Wilson et al. 2007) had a negligible influence on the predicted line profiles of almost all observable transitions, except for C II $\lambda \lambda 3918 / 20$ and 6578/82 $\AA$; see Fig. 7 for an example. The good agreement of abundances derived from these four with other transitions was broken when using the improved Wilson et al. (2007) data, requiring abundance adjustments of up to $\sim 0.3$ dex to match observations. We find that the situation may

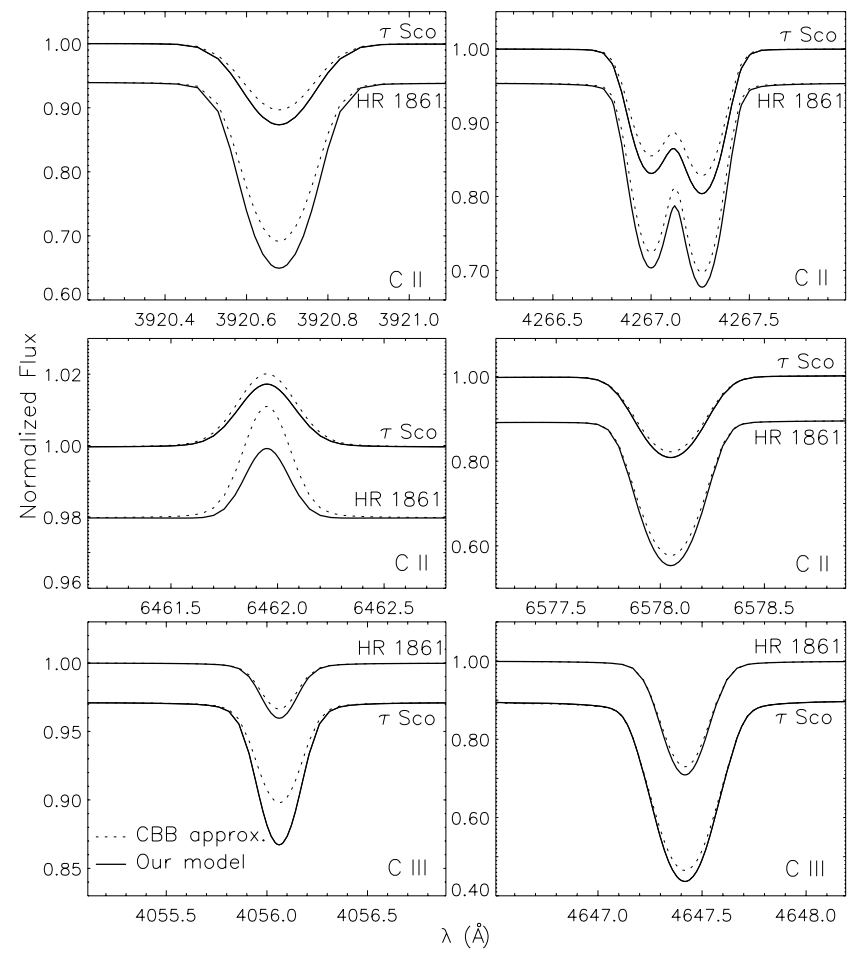

Fig. 8. Comparison of synthetic C II/III line profiles for two of our sample stars using different model atoms. One calculation uses our reference model atom and the other adopts standard approximation formulae for evaluating collisional rates for all transitions.

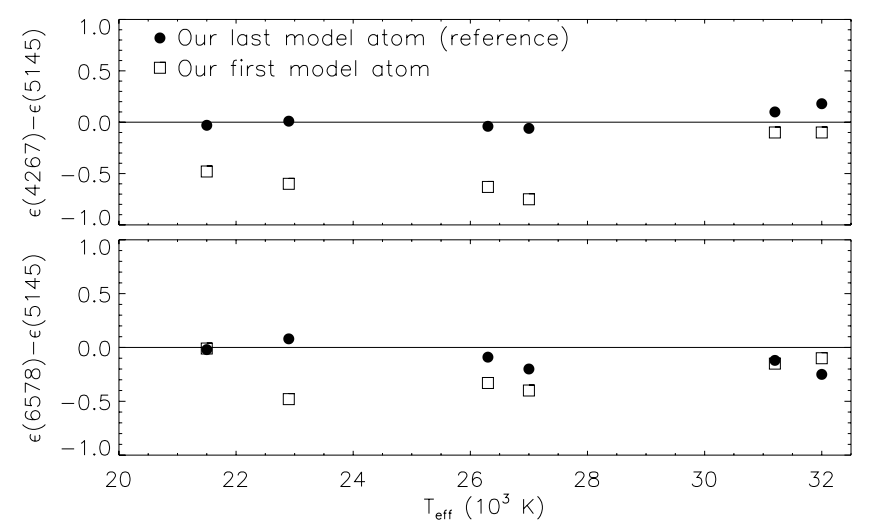

Fig. 9. Abundance differences from the analysis of C II $\lambda \lambda 4267$ and $6578 \AA$ (highly sensitive to the input atomic data) and C II $\lambda 5145 \AA$ ("in LTE"). Displayed are results for our six sample stars, as a function of effective temperature. A comparison of our initial model atom (in particular all radiative transition data for C II from N02a, NP97) and our final model atom after the calibration is shown. See the text for details.

be improved for the stars of our calibration sample by increasing the effective collision strength for the $3 \mathrm{~s}^{2} \mathrm{~S}-3 \mathrm{p}^{2} \mathrm{P}^{\mathrm{o}}$ transition by an empirical factor of two, see Fig. 7. This is larger than the typical uncertainty of such ab-initio data, which amounts to an estimated 10-20\%. However, a closer inspection of the energydependent collision strength for this transition shows that resonances dominate $\Omega$ in the region near threshold. The positions and strengths of the resonances are sensitive to the details of the atomic data calculations, in particular to the assumptions made for constructing the target. Consequently, more comprehensive ab-initio computations are required to investigate this in detail. However, these are beyond the scope of the present work. 

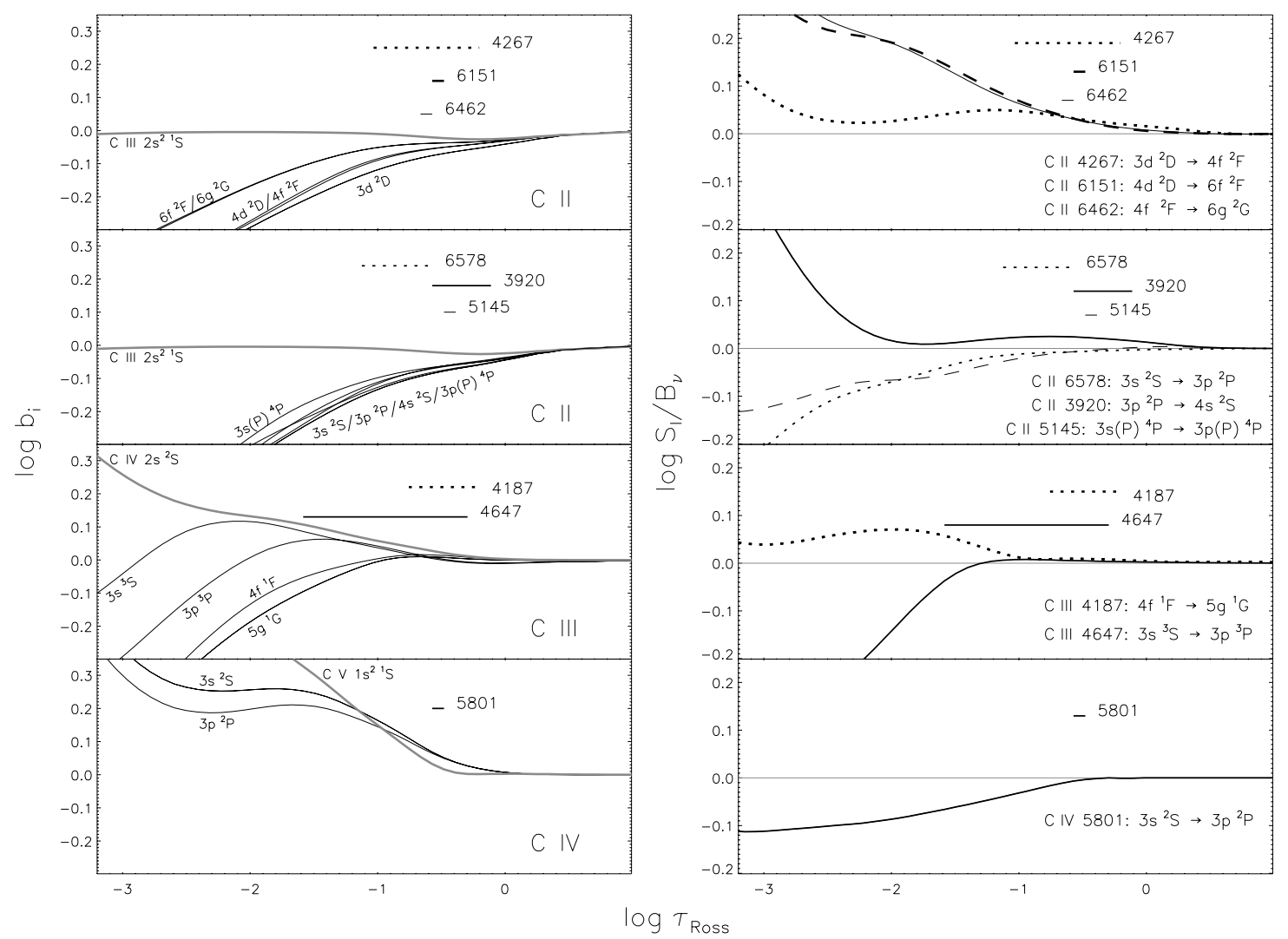

Fig. 10. Departure coefficients $b_{i}$ and ratio of line source-function $S_{1}$ to Planck function $B_{v}$ at line centre as a function of $\tau_{\text {Ross }}$ for selected carbon lines in $\tau$ Sco. The spectral lines are encoded by the different line styles indicating the line-formation depths. Thin grey lines on the right-hand panel correspond to $S_{1}=B_{v}$.

Collisional data from ab-initio calculations are typically available only for transitions between relatively low-lying energy levels. In the case of C II, the dataset is complete for levels up to principal quantum number $n=4$; therefore, for the bulk of the transitions, approximation formulae have to be applied. However, trends and regularities from the ab-initio data may be used to improve on the standard approximations made for these, e.g. dropping the assumption of an energy-independent $\Omega=1$ for the evaluation of the Allen (1973) formula (see Sect. 2.2). In practice, simple approximations are used to describe collisional processes in most of the model atoms available for non-LTE calculations at present. One can ask what the effects on synthetic profiles will be from such a simplification. This is shown in Fig. 8 for selected lines in two of our programme stars. Notable corrections in abundance would be required to reproduce the results from our reference model, an increase in some of the lines but also a decrease in others. Our overall good agreement with observation (Sect. 4) would be destroyed: the non-LTE sensitive lines and the "lines in LTE", which are unaffected by such modifications, would indicate very different abundances. Note that the effects vary from star to star.

We conclude the discussion of the impact of atomic data on line-formation calculations in Fig. 9. Here, we quantify the differences in abundance derived with our initial model atom (built from the available homogeneous set of atomic data, NP97/N02a) and with our model of reference (after the calibration) for the entire sample of stars. Abundances from the strong and non-LTEsensitive C II $\lambda \lambda 4267$ and $6578 \AA$ transitions are compared to abundances derived from the weaker C II $\lambda 5145 \AA$ line, which is almost insensitive to the details of the model atom (i.e. it is "in
LTE"). The relative abundance is displayed as a function of effective temperature for each star (see Sect. 4 for the final results). Pronounced systematic trends exist when the initial model atom is used, correlating with the strength of the lines (i.e. stronger lines show a higher sensitivity to non-LTE effects and therefore to the input atomic data). These trends and abundance differences from C II $\lambda \lambda 4267$ and $6578 \AA$ almost vanish when our model of reference is used. The remaining small differences may be reduced even more when improved atomic data, in particular for collisions involving high-excitation levels, become available. Note, that the C II $26582 \AA$ line (not displayed here) shows a similar trend to that of $\lambda 6578 \AA$. The whole multiplet centred on C II $\lambda 5145 \AA$ also behaves consistently. This kind of test has been made for all C II-IV lines for every set of input atomic data in the calibration process of the model atom. The detailed study of non-LTE and LTE abundances in Sect. 4 helps to identify the lines in LTE for the different atmospheric parameters under analysis. These may provide good starting points for further analyses when one desires to avoid non-LTE effects.

\subsection{Line-formation details}

A closer study of the underlying line-formation processes allows the nature of the non-LTE effects to be understood. This is shown in Fig. 10 for some representative transitions of $\mathrm{C} \mathrm{II} / \mathrm{III} / \mathrm{IV}$ in our programme star $\tau$ Sco. Departure coefficients $b_{i}=n_{i}^{\mathrm{NLTE}} / n_{i}^{\mathrm{LTE}}$ are displayed in the left panel of Fig. 10 for the levels involved in the transitions of interest and the ion ground states. NonLTE departures of the level occupations affect the line source 


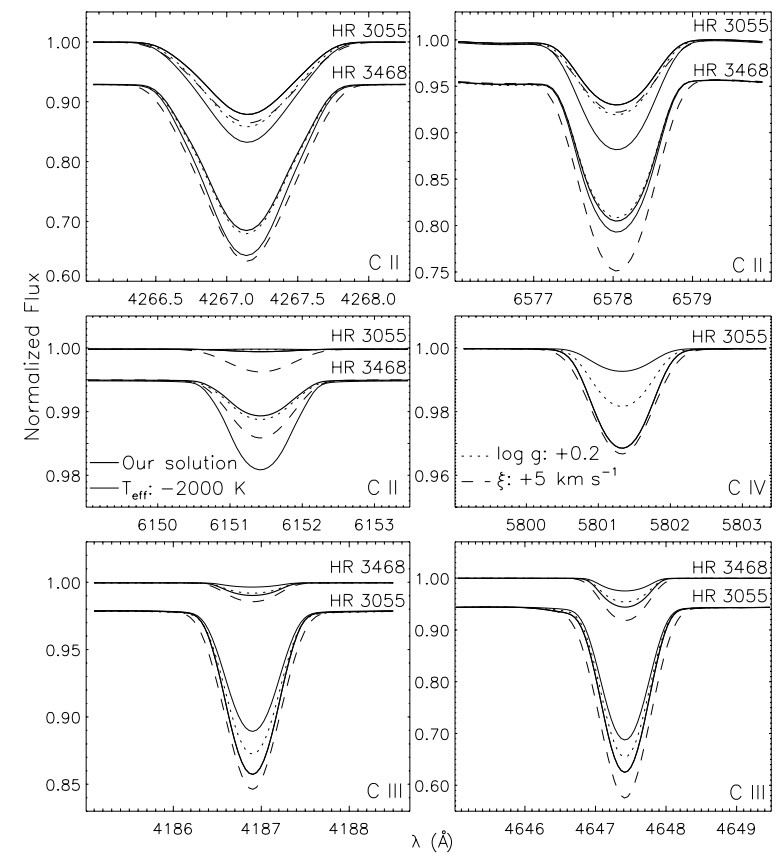

Fig. 11. Sensitivity of selected C II/III/IV lines to atmospheric parameter variations in two giants: HR 3055 (hotter, B0 III) and HR 3468 (cooler, B1.5 III). Our solution corresponds to the final parameters from Table 2 and a constant value of $\varepsilon(\mathrm{C})$ for all lines. The parameter offsets are typical of statistical and systematic uncertainties from published values. The theoretical spectra are convolved with a rotational profile for $v \sin i=20 \mathrm{~km} \mathrm{~s}^{-1}$. Our solution establishes the ionization equilibrium. The star HR 3468 is too cool to show CIV lines.

function $S_{1}$. We recall that the ratio of $S_{1}$ to the Planck function $B_{v}$ (shown for selected transitions in the right panel of Fig. 10) is $S_{1} / B_{v}=\left[\exp \left(h v_{i j} / k T\right)-1\right] /\left[\left(b_{i} / b_{j}\right) \exp \left(h v_{i j} / k T\right)-1\right]$. Here, $S_{1} / B_{v}$ is determined by the ratio of the departure coefficients for the lower and upper levels $(i, j)$. An overpopulation of the upper level relative to the lower (i.e. $S_{1} / B_{v}>1$ ) results in nonLTE weakening of the line and may lead to emission in cases of pronounced overpopulation, while the inverse gives non-LTE strengthening.

The level populations reach detailed equilibrium values deep in the atmosphere, where high collisional rates and small meanfree paths between photon absorptions (both because of the high densities of the plasma) enforce this inner boundary condition. Double-ionized carbon is the main ionization stage at the temperatures of $\tau$ Sco - the C III ground state is close to LTE. Singleionized carbon is overionized at line-formation depths; therefore, the levels are underpopulated relative to LTE, and C IV and the $\mathrm{C}$ V ground state are overpopulated.

In general, level populations in C II depart most from detailed equilibrium in the low-excitation states and approach LTE values gradually with increasing excitation energy, as collisions facilitate coupling with the C III ground state. Therefore, most of the non-LTE-sensitive transitions in C II have upper levels that are overpopulated relative to the lower level, such that the lines experience slight $(\lambda 3920 \AA)$ to notable weakening $(\lambda 4267 \AA)$ relative to LTE and may even turn into emission $(\lambda \lambda 6151,6462 \AA)$. The $\lambda \lambda 6578 / 82 \AA$ doublet experiences non-LTE strengthening for lower effective temperatures, while the lines are found to be close to LTE in hotter objects like $\tau$ Sco. The other observable C II lines arise in the quartet spin system. They are weaker (C II $\lambda 5145 \AA$ is the strongest); i.e., they are formed deeper in
Table 1. Systematic uncertainties in carbon abundances (in dex, relative to our final results, Table 3 ) caused by atmospheric parameter variations and the assumption of LTE for the line-formation calculations.

\begin{tabular}{lcccrr}
\hline \hline & HR 3055 & $\Delta T_{\text {eff }}$ & $\Delta \log g$ & \multicolumn{1}{c}{$\Delta \xi$} & LTE \\
& & $-2000 \mathrm{~K}$ & $+0.2 \mathrm{dex}$ & $+5 \mathrm{~km} \mathrm{~s}^{-1}$ & \\
\hline C II & 4267.2 & -0.33 & -0.11 & -0.16 & -0.40 \\
& 5133.3 & -0.30 & -0.10 & 0.00 & 0.00 \\
& 5143.4 & -0.40 & -0.05 & 0.00 & 0.00 \\
& 5145.2 & -0.32 & -0.09 & -0.02 & 0.00 \\
& 5151.1 & -0.30 & -0.08 & 0.00 & 0.00 \\
& 5662.5 & -0.33 & -0.13 & 0.00 & 0.00 \\
& 6578.0 & -0.40 & -0.15 & -0.10 & -0.01 \\
& 6582.9 & -0.30 & +0.02 & +0.05 & +0.03 \\
C III & 4056.1 & +0.21 & +0.06 & -0.04 & +0.08 \\
& 4162.9 & +0.28 & +0.09 & -0.03 & +0.25 \\
& 4186.9 & +0.35 & +0.15 & -0.08 & +0.07 \\
& 4663.5 & +0.22 & +0.07 & -0.03 & +0.22 \\
& 4665.9 & +0.26 & +0.08 & -0.08 & +0.35 \\
& 5272.5 & +0.16 & +0.01 & 0.00 & 0.00 \\
C IV & 5801.3 & +1.06 & +0.46 & -0.03 & +0.39 \\
& 5811.9 & +1.06 & +0.46 & -0.03 & +0.39 \\
\hline
\end{tabular}

the atmosphere, and their formation involves high-excitation levels that are coupled collisionally at these depths, such that these lines are essentially in LTE. Note that the behaviour of $S_{1} / B_{v}$ for $\lambda 4267 \AA$ is in good agreement with the findings of Sigut (1996). On the other hand, notable differences exist for the $\lambda \lambda 6578 / 82 \AA$ doublet in particular at higher $T_{\text {eff }}$. The reasons for this will be discussed in the next section (but see also Fig. 14).

The C III transitions can also experience both non-LTE weakening (like the strong $\lambda 4187 \AA$ ) and non-LTE strengthening (like the strong triplet 4647-4651 $\AA$ ). The C IV doublet $\lambda \lambda 5801 / 12$, which becomes observable only in our hottest stars, shows a pronounced non-LTE strengthening.

\subsection{Sensitivity of $\varepsilon(C)$ to atmospheric parameter variations}

Spectral lines of carbon, like many other metal lines, can react sensitively to variations in the stellar atmospheric parameters. This property provides us with a powerful tool for the atmospheric parameter and abundance determination, using our iteration scheme. This is a non-trivial and crucial step in the analysis, which must be performed carefully to avoid systematic error. We investigate consequences of systematically biased atmospheric parameters on carbon line profiles and the derived abundances in the following 5 .

The offsets for the parameters (in effective temperature $\Delta T_{\text {eff }}=-2000 \mathrm{~K}$, surface gravity $\Delta \log g=+0.2 \mathrm{dex}$, and microturbulent velocity $\Delta \xi=+5 \mathrm{~km} \mathrm{~s}^{-1}$ ) are representative of systematic discrepancies between our final values and those from previous studies (and also among previous studies). Note that they are much larger than our statistical uncertainties. Such discrepancies may be caused by several factors, among others: I) photometric effective temperature calibrations based on model

\footnotetext{
5 Note that an underestimated source of systematic error in view of line blanketing are abundance 'standards' in the the widely used classical Kurucz (1993a) ODFs, computed on the basis of (scaled) solar abundances according to Anders \& Grevesse (1989). Employing ODFs with appropriately reduced metallicity for the model-atmosphere calculations (see Paper I for a discussion) decreases the atmospheric temperature structure via a backwarming effect, by up to $\sim 500 \mathrm{~K}$ in the line-formation region, i.e. slightly higher than our uncertainties in $T_{\text {eff }}$.
} 


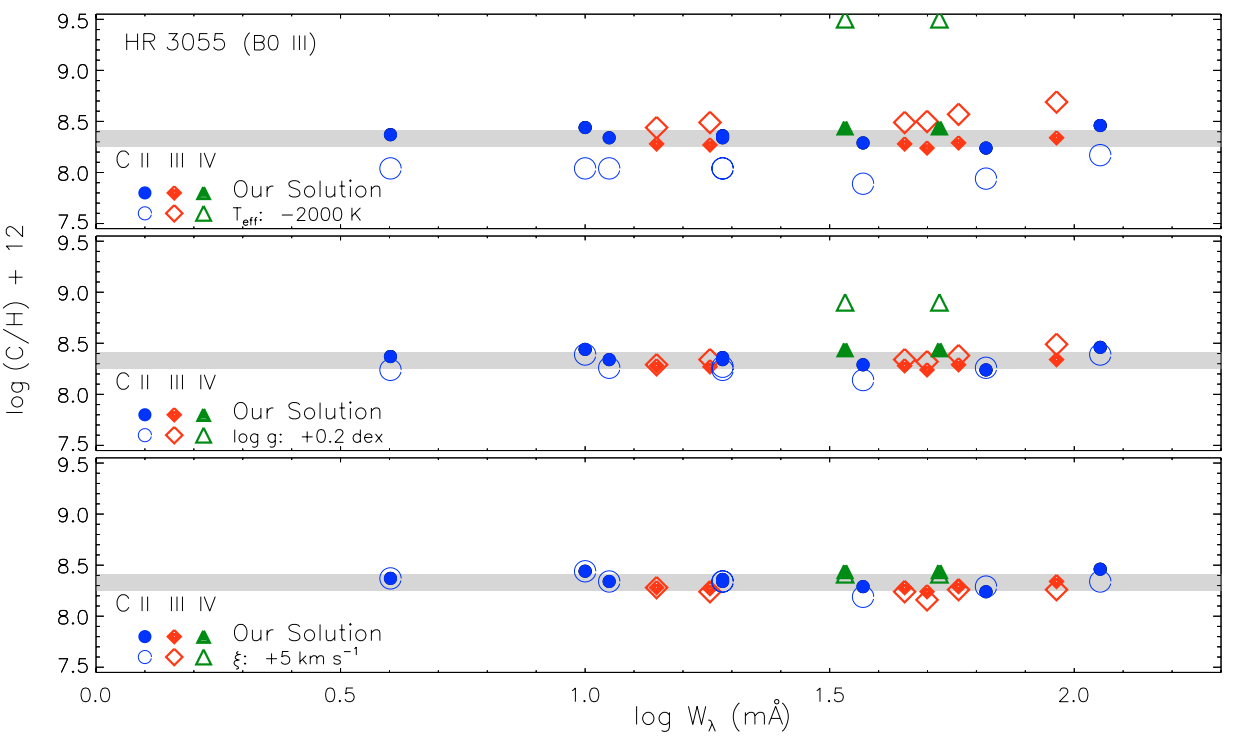

Fig. 12. Sensitivity of carbon abundances to stellar parameter variations: $T_{\text {eff }}$ (upper panel), $\log g$ (mid panel), and microturbulent velocity (lower panel). The offsets of the parameters are displayed in the lower left part of each panel. A wide spread in abundance from individual lines of the three ionization stages results, in particular for variations of $T_{\text {eff }}$ (upper panel). Note also the implications of only using a few lines of one ionization stage for abundance determinations in the presence of systematic errors in the atmospheric parameters. The grey bands correspond to $1 \sigma$-uncertainties of the stellar carbon abundance in our solution (summarised in Table 2).

Table 2. Stellar parameters and carbon abundances of the programme stars.

\begin{tabular}{|c|c|c|c|c|c|c|}
\hline & $\begin{array}{c}\tau \text { Sco } \\
\text { HD } 149438 \\
\end{array}$ & $\begin{array}{c}\text { HR 3055 } \\
\text { HD } 63922 \\
\end{array}$ & $\begin{array}{c}\text { HR 1861 } \\
\text { HD 36591 }\end{array}$ & $\begin{array}{c}\text { HR } 2928 \\
\text { HD } 61068\end{array}$ & $\begin{array}{c}\text { HR 5285 } \\
\text { HD } 122980 \\
\end{array}$ & $\begin{array}{c}\text { HR 3468 } \\
\text { HD 74575 }\end{array}$ \\
\hline & Sco Cen & Field & Ori OB1 & Field & Sco Cen & Field \\
\hline$T_{\text {eff }}(\mathrm{K})$ & $32000 \pm 300$ & $31200 \pm 300$ & $27000 \pm 400$ & $26300 \pm 400$ & $21500 \pm 400$ & $22900 \pm 400$ \\
\hline $\log g$ & $4.30 \pm 0.05$ & $3.95 \pm 0.05$ & $4.12 \pm 0.05$ & $4.15 \pm 0.05$ & $4.20 \pm 0.05$ & $3.60 \pm 0.05$ \\
\hline$\xi\left(\mathrm{km} \mathrm{s}^{-1}\right)$ & $5 \pm 1$ & $8 \pm 1$ & $3 \pm 1$ & $3 \pm 1$ & $3 \pm 1$ & $5 \pm 1$ \\
\hline$v \sin i\left(\mathrm{~km} \mathrm{~s}^{-1}\right)$ & $4 \pm 2$ & $29 \pm 4$ & $12 \pm 1$ & $14 \pm 1$ & $18 \pm 1$ & $11 \pm 2$ \\
\hline$\zeta\left(\mathrm{km} \mathrm{s}^{-1}\right)$ & $4 \pm 2$ & $37 \pm 8$ & & $20 \pm 2$ & & 20 \\
\hline$y(\mathrm{~b}$ & $0.089 \pm 0.01$ & $0.080 \pm 0.01$ & $0.089 \pm 0.01$ & $0.089 \pm 0.01$ & $0.089 \pm 0.01$ & $0.089 \pm 0.01$ \\
\hline$\varepsilon(C)(\operatorname{dex})$ & $8.30 \pm 0.12$ & $8.33 \pm 0.08$ & $8.33 \pm 0.08$ & $8.27 \pm 0.07$ & $8.29 \pm 0.05$ & $8.37 \pm 0.10$ \\
\hline \# C lines & 32 & 19 & 30 & 22 & 22 & 19 \\
\hline
\end{tabular}

atmospheres with insufficient line blanketing, II) spectroscopic ionization equilibria based on predictions of incomplete model atoms, and III) the assumption of LTE for the computation of Balmer line profiles, which may cause a systematic overestimate of $\log g$ by up to 0.2 dex (see Paper I).

Figure 11 shows the sensitivity of selected C II/III/IV lines in two giants of our sample to variations in $T_{\text {eff }}, \log g$, and $\xi$. The profiles accounting for these variations are compared with those computed with our final atmospheric parameters and an averaged carbon abundance of $\varepsilon(\mathrm{C})=8.32$. The sensitivity to parameter variations differs from line to line.

For the hotter stars, the C IV and - when strong enough the C II $\lambda 6151 \AA$ multiplets are ideal indicators of both $T_{\text {eff }}$ and $\log g$, while the rest of the lines are mostly sensitive to changes in $T_{\text {eff }}$ and the strong $\mathrm{C} \mathrm{II} / \mathrm{III}$ lines also to changes in $\xi$. For the cooler stars, the C II $\lambda \lambda 4267$ and $6151 \AA$ multiplets are highly sensitive to variations in $T_{\text {eff }}$ and $\xi$, and the rest of the lines are mostly sensitive to $T_{\text {eff }}$ and the strong lines also to $\xi$.

The response to variations in $\log g$ can be amplified for C II $\lambda \lambda 6578 / 82 \AA$ at higher gravities, because they are formed on the red wing of $\mathrm{H} \alpha$. The local continuum, therefore the lineformation depths, may change as a consequence, affecting the non-LTE effects. A correct treatment of the hydrogen Balmer line-opacity therefore plays an important rôle in this context. Non-LTE effects strengthen the Balmer line wings in particular at higher temperatures (Paper I).

For HR 3055 we have quantified the systematic effects exemplified in Fig. 11 (note that C III $\lambda 4647 \AA$ is blended and
C II $\lambda 6151 \AA$ is too weak in this case) by deriving non-LTE carbon abundances for the modified values of $T_{\text {eff }}, \log g$, and $\xi$ and comparing them to our solution for individual lines. This is summarised in Table 1 and visualised in Fig. 12. Table 1 also shows systematic offsets that arise from the assumption of LTE for the line-formation calculations. This comparison allows us to identify the relative importance of atmospheric parameters/nonLTE effects for some key spectral lines. Note that the C IV lines are extremely sensitive to changes in $T_{\text {eff }}$ and $\log g$ at this temperature $(31200 \mathrm{~K})$, with discrepancies amounting to up to $\sim+1.0$ dex in abundance for $\Delta T_{\text {eff }}=-2000 \mathrm{~K}^{6}$. The $\mathrm{C} \mathrm{II} / \mathrm{III}$ ionization balance is also never established for a variation in these parameters (abundance changes down to -0.40 dex for C II and up to +0.35 dex for C III when compared to our solution). An expected reduction of the abundances from strong lines is obtained for an increased microturbulence. Note that the systematic variations in carbon abundance with $\xi$ for some lines are significant considering the high accuracy we are aiming at, despite smaller effects in general than for $T_{\text {eff }}$ and $\log g$ variations.

The solutions for the modified atmospheric parameters are characterised by a large scatter of $\mathrm{C}$ abundances from individual lines (the statistical 1- $\sigma$ uncertainties increase by up to $\sim 0.4$ dex). Note that variations in $T_{\text {eff }}$ show by far the strongest effects.

We conclude that the use of only a few spectral lines from one ionization stage for $\mathrm{C}$ abundance determinations - which

\footnotetext{
${ }^{6}$ Even larger offsets in $T_{\text {eff }}$ are found with respect to the literature, up to $\Delta T_{\text {eff }} \simeq-4000 \mathrm{~K}$, see Sect. 5 .
} 


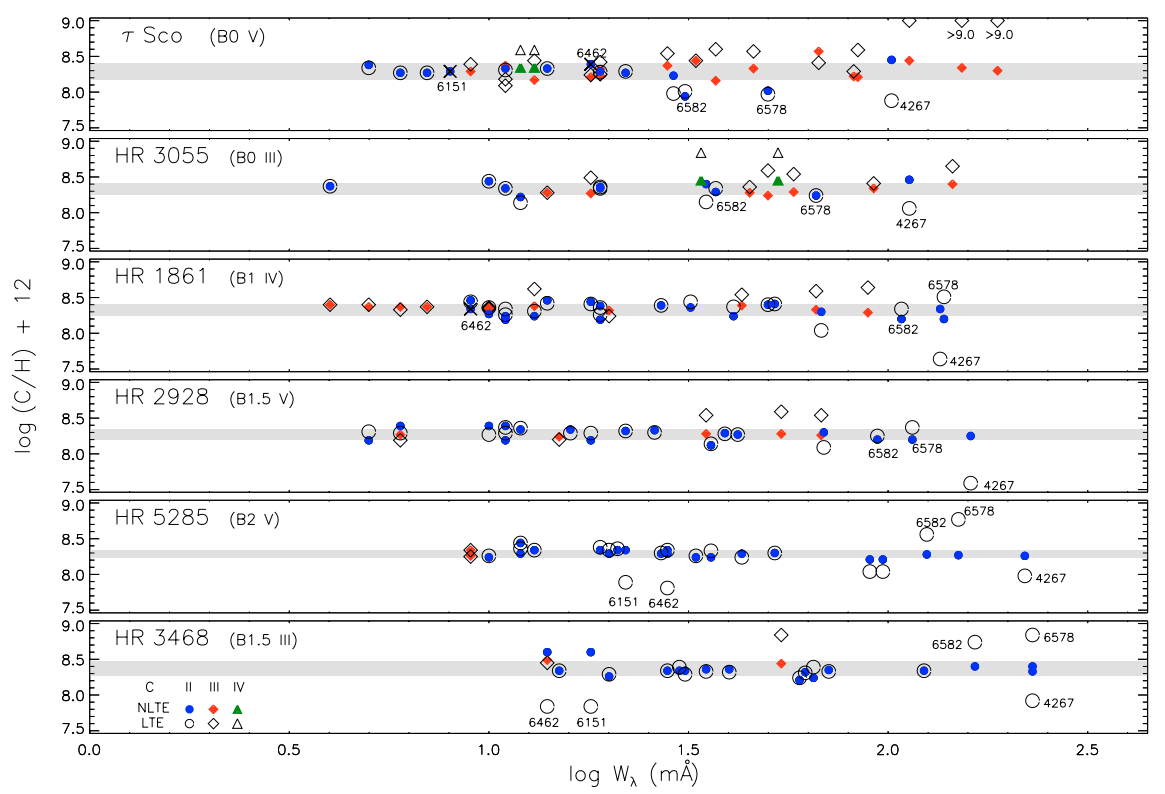

Fig. 13. Non-LTE and LTE abundances derived from line profile fits to individual C II-IV lines in the programme stars as a function of equivalent width. The ID of the stars and the spectral classification is given in the upper left corner of each panel. The grey rectangles correspond to $1 \sigma$-uncertainties of the stellar carbon abundance from the line-to-line scatter. Identification of lines with high sensitivity to non-LTE effects is displayed. Emission lines are marked by crosses (C II $\lambda \lambda 6151$ and $6462 \AA$ in $\tau$ Sco and $\lambda 6462 \AA$ in HR 1861): LTE calculations are not able to reproduce them even qualitatively. is common practice in the literature - may have serious implications for the final accuracy. Possible systematic discrepancies, as indicated here, may remain unrecognised. Moreover, the opportunity to improve on the atmospheric parameter determination by establishing the highly parameter-sensitive ionization balance may be missed in such cases. This high sensitivity of the carbon lines to atmospheric parameter variations can be used as an important tool for precise quantitative spectral analyses of this kind of star when applying the calibrated model atom in the future.

Some lines are practically unaffected by non-LTE effects, as indicated by Table 1 (see also Sect. 3.2). This implies that they are almost insensitive to any reasonable choice of model atom. On the other hand, they are highly sensitive to the choice of atmospheric parameters. This property is highly useful for the model atom calibration because it helps to disentangle effects due to non-LTE from those due to inaccuracies in the stellar parameters, facilitating a reduction of systematic errors. Typical systematic uncertainties in the atmospheric parameters can have a similar or even stronger impact on the carbon abundance determination than a neglect of non-LTE effects.

\section{Results}

Accurate atmospheric parameters and carbon abundances are derived from line-profile fitting by $\chi^{2}$ minimization, which puts tighter constraints than only matching equivalent widths. These are summarised in Table 2 for our six programme stars (as obtained from our iterative process, Fig. 1). The uncertainties of $T_{\text {eff }}$ and $\log g$ are estimated from the extremely sensitive carbon ionization equilibrium. They are lower for hotter stars because of the additional restrictions imposed by the presence of lines from three ionization stages. For velocities, we provide the $1 \sigma$-uncertainties from the analysis of the entire line ensemble. Projected rotational velocity and (radial-tangential) macroturbulence (Gray 1992, p. 407ff.) were simultaneously derived allowing for small line-to-line variations in order to obtain an optimum fit (see Ryans et al. 2002, for the case of B supergiants).

We should emphasise that the final set of atmospheric parameters as derived from the $\mathrm{C} \mathrm{II/III/IV} \mathrm{ionization} \mathrm{balance} \mathrm{agrees}$ with those from our previous quantitative analyses of these stars. In particular, a simultaneous match is achieved for I) the $\mathrm{H}$ and He lines in the visual and (where available) in the nearIR, including the He I/II ionization equilibrium in the hotter stars (Paper I, also the source of helium abundance $y$ ) and II) the spectral energy distributions from the UV to the near IR (Paper II). A similar degree of consistency is typically not obtained in comparable studies of early-type stars.

A large quantity of carbon lines is analysed for the first time, giving consistent abundances for all of them. The excellent quality of the observed spectra combined with our improved analysis technique allow us to achieve such precise results. The $1 \sigma$-uncertainties from the line-to-line scatter are typically of the order of $0.05-0.10$ dex. We estimate the systematic uncertainties due to remaining errors in atmospheric parameters and atomic data to be of the order of $0.10-0.15$ dex, taking Table 1 and our experiences from Sect. 3.2 as a guideline.

Details on the analysis of individual lines can be found in Table 3. This summarises line identifications, level designations, $\log g f$ values, excitation potential of the lower level $\chi_{l}$, and for each star equivalent widths $W_{\lambda}$ and the derived non-LTE and LTE abundances. Note that we analyse as many lines as possible per star, excluding features with strong blends by other chemical species. This helps us to understand the behaviour of each line and the quality of our modelling at different temperatures and gravities (see the previous section).

The high quality of our line fits to the observed spectrum of the programme stars is demonstrated in Figs. 17-22, available online, for almost all the analysed transitions. Of central importance is that the abundances derived from the individual lines show a small scatter in each sample star. Good fits to individual lines can almost always be obtained; however, this does not imply consistency in the entire analysis. An example are the abundances from Fig. 12, which were also derived from highquality line fits, but which show inconsistencies, expressed as a large line-to-line scatter, nonetheless. Another example from many other studies are discrepant lines that are excluded from the analysis in order to reduce the statistical uncertainties. The present work improves on this because the underlying physics is solved in a more consistent way.

We find a single exception to our overall good line fits: the doublet C II $\lambda \lambda 4267.0 / 2 \AA$ in $\tau$ Sco, where the fine-structure components are resolved (Fig. 17). The synthetic profile is 
Table 3. Carbon abundance analysis for the programme stars.

\begin{tabular}{|c|c|c|c|c|c|c|c|c|c|c|c|c|c|c|c|c|c|c|c|c|c|c|}
\hline \multirow[b]{2}{*}{ Ion } & \multirow[b]{2}{*}{$\lambda(\AA)$} & \multirow{2}{*}{\multicolumn{2}{|c|}{$1-u$}} & \multirow[b]{2}{*}{$\log g f$} & \multirow[b]{2}{*}{$\chi_{l}(\mathrm{eV})$} & \multicolumn{3}{|c|}{$\bar{\tau} \tau \mathrm{Sco}$} & \multicolumn{3}{|c|}{ HR 3055 } & \multicolumn{3}{|c|}{$\overline{~ H R ~} 1861$} & \multicolumn{3}{|c|}{ HR 2928} & \multicolumn{2}{|c|}{ HR 5285} & \multicolumn{3}{|c|}{ HR 3468 } \\
\hline & & & & & & $W_{\lambda}$ & NLTE & LTE & $W_{\lambda}$ & NLTE & LTE & $W_{\lambda}$ & NLTE & E LTE & $W_{\lambda}$ & NLTE & LTE & $W_{\lambda}$ NLT & LTE & $W_{\lambda} \mathrm{l}$ & NLTE & E LTE \\
\hline \multirow[t]{21}{*}{$\mathrm{C}_{\text {II }}$} & 3919.0 & $3 p^{2} P$ & $-4 s^{2} S$ & $-0.53^{B}$ & 16.33 & & $\mathrm{bl}$ & & & $\mathrm{bl}$ & & & bl & & & $\mathrm{bl}$ & & 908.21 & 8.04 & & $\mathrm{bl}$ & \\
\hline & 3920.6 & & $"$ & $-0.23^{B}$ & 16.33 & 29 & 8.23 & 7.98 & 35 & 8.40 & 8.15 & 68 & 8.30 & 8.04 & 69 & 8.30 & 8.09 & 978.21 & 8.04 & 123 & 8.34 & 8.34 \\
\hline & $4267.0 / 2$ & $3 d^{2} D$ & $-4 \mathrm{f}^{2} \mathrm{~F}^{\circ}$ & $0.56 / 0.74^{C+}$ & 18.04 & 102 & 8.45 & 7.88 & 113 & 8.46 & 8.06 & 135 & 8.34 & 7.64 & 161 & 8.25 & 7.59 & 2208.20 & 7.98 & 230 & 8.33 & 7.92 \\
\hline & $5133.0 / 3$ & $\left({ }^{3} \mathrm{P}^{\circ}\right) 3 \mathrm{~s}^{4} \mathrm{P}^{\circ}$ & $-\left({ }^{3} \mathrm{P}^{\circ}\right) 3 \mathrm{p}^{4} \mathrm{P}$ & $-0.21 /-0.18^{B}$ & 20.70 & 19 & 8.29 & 8.28 & 19 & 8.34 & 8.34 & 52 & 8.41 & 8.41 & 42 & 8.27 & 8.27 & 528.30 & 8.30 & 71 & 8.35 & 8.33 \\
\hline & 5137.3 & & $"$ & $-0.91^{B}$ & 20.70 & & $\ldots$ & & & $\ldots$ & & 9 & 8.46 & 8.44 & 6 & 8.39 & 8.29 & 128.44 & 8.44 & & $\ldots$ & \\
\hline & 5139.2 & & $"$ & $-0.71^{B}$ & 20.70 & 5 & 8.38 & 8.34 & & $\ldots$ & & 14 & 8.46 & 8.42 & 10 & 8.39 & 8.27 & 138.34 & 8.34 & 15 & 8.34 & 8.34 \\
\hline & 5143.4 & & $"$ & $-0.22^{B}$ & 20.70 & 11 & 8.33 & 8.31 & 10 & 8.44 & 8.44 & 27 & 8.39 & 8.39 & 26 & 8.33 & 8.30 & $28 \quad 8.34$ & 8.34 & 35 & 8.36 & 8.33 \\
\hline & 5145.2 & & $"$ & $0.19^{B}$ & 20.71 & 22 & 8.27 & 8.29 & 19 & 8.36 & 8.36 & 50 & 8.40 & 8.40 & 39 & 8.29 & 8.28 & $43 \quad 8.29$ & 8.24 & 62 & 8.32 & 8.31 \\
\hline & 5151.1 & & $"$ & $-0.18^{B}$ & 20.71 & 14 & 8.33 & 8.33 & 11 & 8.34 & 8.34 & 32 & 8.36 & 8.44 & 22 & 8.32 & 8.32 & $27 \quad 8.29$ & 8.30 & 40 & 8.36 & 8.32 \\
\hline & 5648.1 & $\left({ }^{3} \mathrm{P}^{\circ}\right) 3 \mathrm{~s}^{4} \mathrm{P}^{\circ}$ & $-\left({ }^{3} \mathrm{P}^{\circ}\right) 3 \mathrm{p}^{4} \mathrm{~S}$ & $-0.42^{B}$ & 20.70 & 6 & 8.27 & 8.27 & & $\ldots$ & & 18 & 8.44 & 8.41 & 11 & 8.39 & 8.37 & 198.34 & 8.38 & 28 & 8.34 & 8.34 \\
\hline & 5662.5 & & $"$ & $-0.25^{B}$ & 20.71 & 7 & 8.27 & 8.27 & 4 & 8.37 & 8.37 & 19 & 8.38 & 8.36 & 16 & 8.34 & 8.29 & 218.34 & 8.36 & 31 & 8.34 & 8.29 \\
\hline & 6578.0 & $3 s^{2} S$ & $-3 p^{2} \mathrm{P}^{\circ}$ & $-0.03^{N}$ & 14.45 & 50 & 8.02 & 8.01 & 66 & 8.24 & 8.24 & 138 & 8.20 & 8.51 & 115 & 8.20 & 8.37 & 1508.27 & 8.77 & 230 & 8.40 & 8.84 \\
\hline & 6582.9 & & " & $-0.33^{N}$ & 14.45 & 31 & 7.94 & 7.97 & 37 & 8.29 & 8.34 & 108 & 8.20 & 8.34 & 94 & 8.20 & 8.25 & $125 \quad 8.28$ & 8.56 & 165 & 8.40 & 8.74 \\
\hline & 6779.9 & $\left({ }^{3} \mathrm{P}^{\circ}\right) 3 \mathrm{~s}^{4} \mathrm{P}^{\circ}$ & $-\left({ }^{3} \mathrm{P}^{\circ}\right) 3 \mathrm{p}^{4} \mathrm{D}$ & $0.02^{B}$ & 20.70 & & $\ldots$ & & & $\ldots$ & & 19 & 8.19 & 8.26 & 36 & 8.12 & 8.14 & 338.24 & 8.26 & 60 & 8.21 & 8.24 \\
\hline & 6780.6 & & $"$ & $-0.38^{B}$ & 20.70 & & $\ldots$ & & & $\ldots$ & & 11 & 8.19 & 8.26 & & bl & & $\mathrm{bl}$ & & & $\mathrm{bl}$ & \\
\hline & 6783.1 & & $"$ & $0.30^{B}$ & 20.71 & & $\ldots$ & & 12 & 8.22 & 8.14 & 41 & 8.24 & 8.37 & 5 & 8.19 & 8.31 & 368.24 & 8.33 & 65 & 8.24 & 8.39 \\
\hline & 6787.2 & & $"$ & $-0.38^{B}$ & 20.70 & & $\ldots$ & & & $\ldots$ & & 11 & 8.24 & 8.34 & 11 & 8.19 & 8.29 & 128.29 & 8.36 & 30 & 8.34 & 8.39 \\
\hline & 6791.5 & & $"$ & $-0.27^{B}$ & 20.70 & & $\ldots$ & & & $\ldots$ & & 13 & 8.24 & 8.31 & 18 & 8.19 & 8.29 & 208.29 & 8.34 & 20 & 8.26 & 8.29 \\
\hline & 6800.7 & & $"$ & $-0.34^{B}$ & 20.71 & & $\ldots$ & & & $\ldots$ & & 10 & 8.27 & 8.36 & 12 & 8.34 & 8.36 & 108.24 & 8.26 & & $\ldots$ & \\
\hline & $6151.3 / 5$ & $4 d^{2} D$ & $-6 f^{2} \mathrm{~F}^{\circ}$ & $-0.15 / 0.02^{N}$ & 20.84 & -8 & 8.29 & $\mathrm{XX}$ & & $\ldots$ & & & $\ldots$ & & & $\ldots$ & & 228.34 & 7.89 & 18 & 8.60 & 7.84 \\
\hline & 6461.9 & $4 \mathrm{f}^{2} \mathrm{~F}^{\circ}$ & $-6 g^{2} G$ & $0.42^{N}$ & 20.95 & -18 & 8.39 & $\mathrm{XX}$ & & $\ldots$ & & -9 & 8.34 & $\mathrm{XX}$ & & $\ldots$ & & $28 \quad 8.29$ & 7.81 & 14 & 8.60 & 7.84 \\
\hline \multirow[t]{17}{*}{$\mathrm{C}$ III } & 4056.1 & $4 d^{1} D^{\circ}$ & $-5 f^{1} \mathrm{~F}$ & $0.27^{B}$ & 40.20 & 33 & 8.44 & 8.44 & 45 & 8.28 & 8.36 & 10 & 8.36 & 8.36 & 6 & 8.27 & 8.19 & $\ldots$ & & & $\ldots$ & \\
\hline & 4152.5 & $\left({ }^{3} \mathrm{P}^{\circ}\right) 3 \mathrm{p}^{3}$ & $D-5 f^{3} F^{\circ}$ & $-0.11^{C+}$ & 40.06 & 28 & 8.37 & 8.54 & & $\mathrm{bl}$ & & 7 & 8.37 & 8.37 & & $\ldots$ & & $\ldots$ & & & $\ldots$ & \\
\hline & 4162.9 & & $"$ & $-0.84^{C+}$ & 40.06 & 46 & 8.33 & 8.57 & 58 & 8.29 & 8.54 & & bl & & & $\mathrm{bl}$ & & $\ldots$ & & & $\ldots$ & \\
\hline & 4186.9 & $4 \mathrm{f}^{1} \mathrm{~F}$ & $-5 g^{1} \mathrm{G}$ & $0.92^{B}$ & 40.01 & 67 & 8.57 & 8.41 & 92 & 8.34 & 8.41 & 20 & 8.32 & 8.24 & 15 & 8.24 & 8.20 & $\cdots$ & & 14 & 8.49 & 8.45 \\
\hline & 4515.8 & $4 p^{3} \mathrm{P}$ & $-5 s^{3} s$ & $-0.28^{B}$ & 39.40 & 18 & 8.21 & 8.24 & & $\mathrm{bl}$ & & 4 & 8.40 & 8.40 & & $\ldots$ & & $\ldots$ & & & $\ldots$ & \\
\hline & 4516.8 & & " & $-0.06^{B}$ & 39.40 & 19 & 8.23 & 8.25 & & bl & & 6 & 8.37 & 8.33 & & $\ldots$ & & $\ldots$ & & & $\ldots$ & \\
\hline & 4647.4 & $3 s^{3} s$ & $-3 p^{3} P$ & $0.07^{B+}$ & 29.53 & 188 & 8.30 & $\geq 9.0$ & & bl & & 89 & 8.29 & 8.64 & 68 & 8.26 & 8.54 & 98.26 & 8.25 & 54 & 8.44 & 8.84 \\
\hline & 4650.2 & & " & $-0.15^{B+}$ & 29.53 & 153 & 8.34 & $\geq 9.0$ & & bl & & 66 & 8.33 & 8.59 & 54 & 8.28 & 8.59 & 98.34 & 8.34 & & bl & \\
\hline & 4651.5 & & $"$ & $-0.63^{B+}$ & 29.53 & 113 & 38.44 & 9.0 & & $\mathrm{bl}$ & & 43 & 8.39 & 8.54 & 35 & 8.28 & 8.54 & $\mathrm{bl}$ & & & $\mathrm{bl}$ & \\
\hline & 4663.5 & $\left({ }^{3} \mathrm{P}^{\circ}\right) 3 \mathrm{~s}^{3} \mathrm{P}^{\circ}$ & $-\left({ }^{3} \mathrm{P}^{\circ}\right) 3 \mathrm{p}^{3} \mathrm{P}$ & $-0.61^{B}$ & 38.22 & 13 & 8.17 & 8.44 & 18 & 8.27 & 8.49 & 5 & 8.37 & 8.40 & & $\ldots$ & & $\ldots$ & & & $\ldots$ & \\
\hline & 4665.9 & & $"$ & $-0.03^{B}$ & 38.22 & 37 & 8.16 & 8.60 & 50 & 8.24 & 8.59 & 13 & 8.38 & 8.62 & & $\ldots$ & & $\ldots$ & & & $\ldots$ & \\
\hline & 5253.6 & $\left({ }^{3} \mathrm{P}^{\circ}\right) 3 \mathrm{~s}^{3} \mathrm{P}^{\circ}$ & $-\left({ }^{3} \mathrm{P}^{\circ}\right) 3 \mathrm{p}^{3} \mathrm{~S}$ & $-0.71^{B}$ & 38.22 & 9 & 8.29 & 8.39 & & $\ldots$ & & & $\ldots$ & & & $\ldots$ & & $\ldots$ & & & $\ldots$ & \\
\hline & 5272.5 & & " & $-0.49^{B}$ & 38.23 & 19 & 8.32 & 8.42 & 14 & 8.28 & 8.28 & & $\ldots$ & & & $\ldots$ & & $\ldots$ & & & $\ldots$ & \\
\hline & 5695.9 & $3 p^{1} \mathrm{P}$ & $-3 d^{1} D$ & $0.02^{B}$ & 32.10 & 82 & 8.22 & 8.29 & & $\mathrm{bl}$ & & 10 & 8.34 & 8.34 & & $\mathrm{bl}$ & & $\ldots$ & & & $\ldots$ & \\
\hline & 6731.0 & $\left({ }^{3} \mathrm{P}^{\circ}\right) 3 \mathrm{~s}^{3} \mathrm{P}^{\circ}$ & $-\left({ }^{3} \mathrm{P}^{\circ}\right) 3 \mathrm{p}^{3} \mathrm{D}$ & $-0.29^{B}$ & 38.22 & 11 & 8.37 & 8.18 & & $\ldots$ & & & $\ldots$ & & & $\ldots$ & & $\ldots$ & & & $\ldots$ & \\
\hline & 6744.3 & & $"$ & $-0.02^{B}$ & 38.23 & 11 & 8.33 & 8.09 & & $\ldots$ & & & $\ldots$ & & & $\ldots$ & & $\ldots$ & & & $\ldots$ & \\
\hline & 8500.3 & $3 s^{1} s$ & $-3 p{ }^{1} \mathrm{P}$ & $-0.48^{B}$ & 30.64 & 84 & 8.21 & 8.59 & 145 & 8.40 & 8.65 & & $\mathrm{bl}$ & & & $\mathrm{bl}$ & & $\ldots$ & & & $\ldots$ & \\
\hline \multirow[t]{2}{*}{ C IV } & 5801.3 & $3 \mathrm{~s}^{2} \mathrm{~S}$ & $-3 \mathrm{~s}^{2} \mathrm{P}^{\circ}$ & $-0.19^{A}$ & 37.55 & 13 & 8.34 & 8.59 & 53 & 8.45 & 8.84 & & $\ldots$ & & & $\ldots$ & & $\ldots$ & & & $\ldots$ & \\
\hline & 5811.9 & $3 \mathrm{~s}^{2} \mathrm{~S}$ & $-3 \mathrm{~s}^{2} \mathrm{P}^{\circ}$ & $-0.49^{A}$ & 37.55 & 12 & 8.34 & 8.59 & 34 & 8.45 & 8.84 & & $\ldots$ & & & $\ldots$ & & $\ldots$ & & & $\ldots$ & \\
\hline
\end{tabular}

bl: blend with other metal line; ... : too weak or absent; XX: LTE does not reproduce the emission; $\log g f$-values in the formal solution: Wiese et al. (1996, uncertainties within: $3 \%$ A, 10\% B, 25\% C) and Nahar (2002a, uncertainties within 10-25\% N).

slightly broader than the observed one, even when neglecting micro- and macroturbulent broadening. Such a detail cannot be observed in the other stars because of their higher $v \sin i$.

The data from Table 3 are visualised in Fig. 13, where abundances are shown as a function of $W_{\lambda}$. Excellent consistency is found in the case of the non-LTE analysis while the quality of the results is considerably degraded in LTE. Note that the subset of the strong lines provides non-LTE abundances that are in good agreement with those derived from the entire sample of lines. This proves that our reference model atom is well suited to analyses of low-S/N spectra and fast-rotating stars, where only the strongest lines are measurable and where such a consistency check is not feasible. Note also that the non-LTE effects differ from line to line and also from star to star.

As the carbon abundances presented here are derived via line-profile fitting of our synthetic spectra, they do not depend on equivalent width measurements. However, careful determinations of $W_{\lambda}$ via direct integration, cross-checked by Voigt-profile fitting, were performed to allow a visualisation like in Fig. 13. Comparisons between both methods for $W_{\lambda}$ measurements can give discrepancies up to $10-20 \%$ in the worst case, mostly for weak lines in spectral regions where the continuum is difficult to be set, the $\mathrm{S} / \mathrm{N}$ is slightly degraded or there are partial blends with other lines.

One case requires further discussion: the C II $\lambda \lambda 6578 / 82 \AA$ doublet in $\tau$ Sco. The remaining problems may be an artifact from the data reduction. The location of the red wing of $\mathrm{H} \alpha$ coincides with a bad column of the CCD of FEROS (the spectral orders are oriented along columns in this spectrograph). A perfect correction for this cannot be provided. Different wavelengths are affected, depending on the radial velocity shifts of the object. Note that the region around C II $\lambda \lambda 6578 / 82 \AA$ doublet has been normalised relative to the local continuum in Fig. 17.

Finally, even when non-LTE-insensitive lines are considered in the analysis, it is mandatory to verify that the ionization balance has been established, this in order to avoid systematic errors in the atmospheric parameters (in particular $T_{\text {eff }}$ ) and therefore in abundance. It is recommended that such lines in LTE be used for abundance analyses when an optimised model atom is not available and the $\mathrm{S} / \mathrm{N}$ of the observed spectra is high enough to measure them. Table 3 may act as a guideline to identify appropriate transitions, depending on atmospheric parameters. 


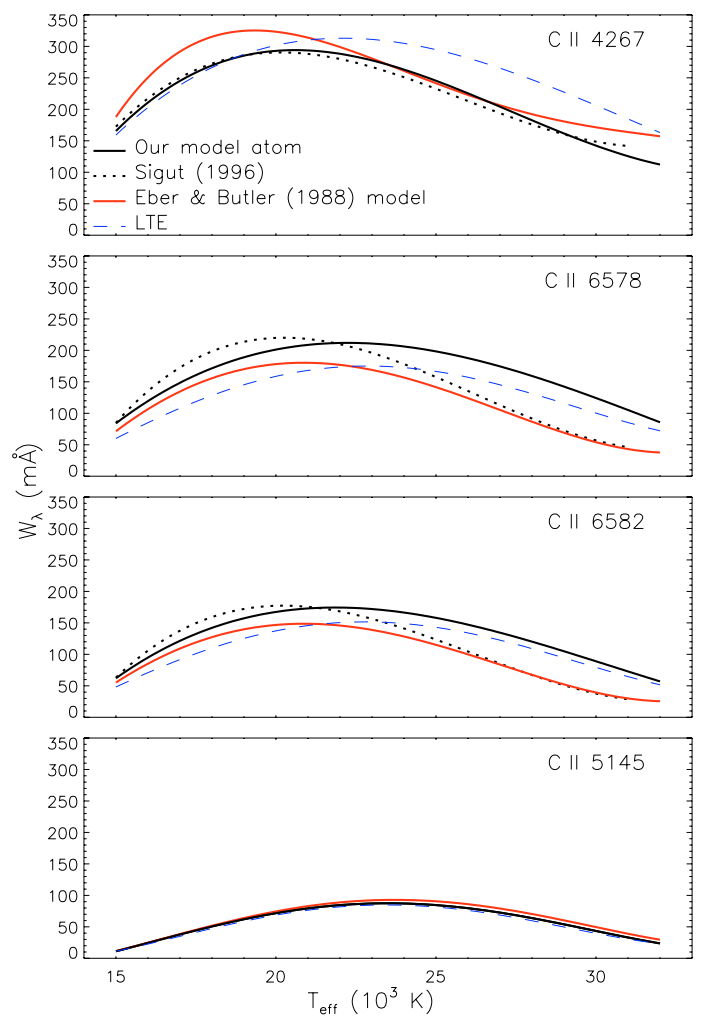

Fig. 14. Predicted equivalent widths of some $\mathrm{C}$ II lines from different approaches as a function of $T_{\text {eff }}$. All calculations were performed for $\log g=4.0, \xi=5 \mathrm{~km} \mathrm{~s}^{-1}$ and $\varepsilon(\mathrm{C})=8.55$, in order to facilitate a comparison with Sigut (1996).

\section{Comparison with previous work}

We concentrate here on the main sources of systematic uncertainties that can bias abundance analyses, as identified in Sects. 3.2 and 3.4: atomic data, i.e. different model atoms, and stellar parameters, i.e. in particular $T_{\text {eff }}$ scales. A more comprehensive comparison is too complex to be performed because of the large number of variables involved, which in some cases are not even documented. This includes numerous factors related to observation (e.g. quality of the analysed spectra, continuum rectification, equivalent width measurements, line blends), model atmospheres (e.g. codes, abundance standards and linelists considered for line blanketing) and line-formation calculations (e.g. handling of line blocking, oscillator strengths, line broadening). A comparison of our results on carbon abundances in the solar neighbourhood with previous studies will be made in an extra section, because of the wider implications.

\subsection{Predictions from different non-LTE model atoms}

We computed non-LTE line profiles based on the present model atom of reference and the Eber \& Butler (1988, EB88) model and have compared them with non-LTE data from Sigut (1996) and our LTE results in Fig. 14. All models are calculated for the same set of atmospheric parameters and carbon abundances. Note that only one fundamental difference exists: our computations account for non-LTE populations for hydrogen while Sigut assumes LTE. This is one of the reasons why Sigut (1996) found problems in reproducing observed trends for the C II $\lambda \lambda 6578 / 82 \AA$ doublet at $T_{\text {eff }}>25000 \mathrm{~K}: \mathrm{H} \alpha$ was assumed to be formed in LTE in that work.

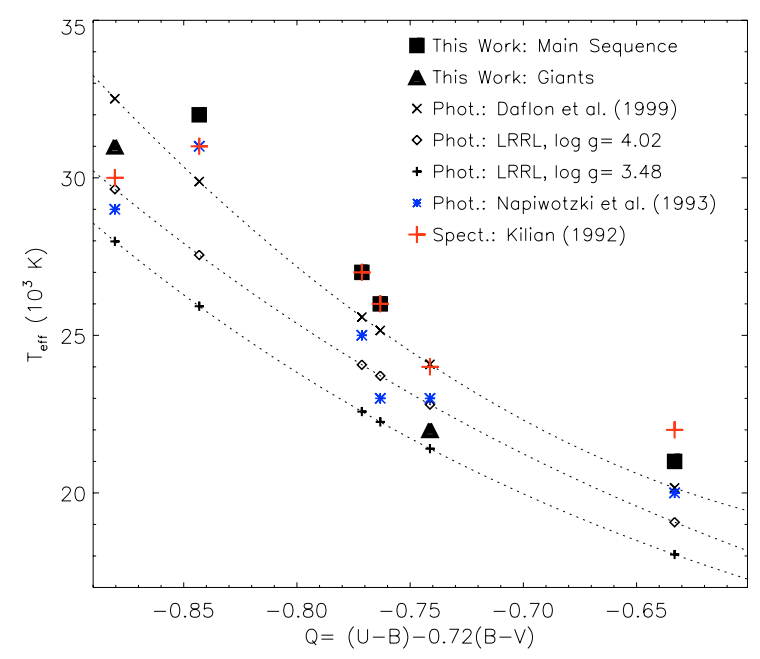

Fig. 15. Comparison of our final $T_{\text {eff }}$ for the sample stars with values derived from photometric calibrations: $T_{\text {eff }}(Q)$ (Daflon et al. 1999; Lyubimkov et al. 2002: LRRL) and $T_{\text {eff }}([u-b])$ (Napiwotzki et al. 1993). Photometric indices are computed from SIMBAD data. Spectroscopic temperatures according to Kilian (1992) are also displayed.

We obtained consistent abundances from the application of our model atom of reference to observations for the C II $\lambda 4267 \AA$ transition, as shown in Sect. 4. Consequently, the good agreement with Sigut's predictions indicates that his model atom is also highly useful for abundance determinations from this line. Note that Sigut compares his results (for $\varepsilon(C)=8.55$ ) only qualitatively with observed equivalent widths from different sources, using stellar parameters derived in one of those studies or from a photometric $T_{\text {eff-calibration. A reduction of carbon abundance }}$ and an improved determination of atmospheric parameters as proposed in the present work may bring observation in much better agreement with his predictions (his Fig. 1). In the region $\sim 22000 \mathrm{~K} \leq T_{\text {eff }} \leq 28000 \mathrm{~K}$, good agreement is also found with the predictions of the EB88 model atom. However, this model predicts equivalent widths that are too large and therefore lower abundances outside this region.

Our analysis gives abundances from the $\lambda \lambda 6578 / 82 \AA$ doublet consistent with those from other lines ( $\tau$ Sco may be an exception, as discussed above). The trends predicted with our model atom of reference differ from the three other model calculations. Sigut's and EB88's non-LTE predictions agree qualitatively up to $T_{\text {eff }} \sim 22000 \mathrm{~K}$, Sigut's model indicating larger and EB88 smaller equivalent widths, respectively. However, our model shows non-LTE strengthening throughout, approaching LTE at the high $T_{\text {eff }}$-limit, while the two other models imply pronounced non-LTE weakening at $T_{\text {eff }} \gtrsim 25000 \mathrm{~K}$. Note that this doublet experiences significant non-LTE effects only when the lines are strong, e.g. for high values of carbon abundance, such as those discussed in Fig. 14. The non-LTE effects are abundance-dependent, they are reduced with decreasing abundance. Finally, the predicted equivalent widths for the widely used C II multiplet including $\lambda 5145 \AA$ are practically independent of the model assumptions. The lines are close to LTE. Any inconsistencies of carbon abundances based on this multiplet have to be related to other effects, but not to the choice of the model atom. This multiplet, of the quartet spin system, was not analysed by Sigut. 


\subsection{Effective temperatures}

Abundance determinations can be systematically biased even when a realistic non-LTE model atom is available. The atmospheric parameters need to be derived accurately as well. We have quantified the effects of inappropriately chosen atmospheric parameters in Sect. 3.4. Largely discrepant abundances are found from different ionization stages in particular for inaccurate effective temperatures. We were confronted with this from the beginning of our work, when adopting effective temperatures derived from common photometric calibrations. It was not possible to establish the ionization equilibrium of $\mathrm{C} \mathrm{II} / \mathrm{III}$ even for the lines in LTE in that case. This motivated us to use an iterative approach to constrain all variables that are involved in the analysis simultaneously and in a self-consistent way.

The final values for $T_{\text {eff }}$ are compared with other derivations in Fig. 15. The comparison concentrates on widely used photometric $T_{\text {eff }}$-calibrations, which provide a fast determination of atmospheric parameters, allowing the analysis of larger samples of stars to be performed. This includes methods using broad-band Johnson photometry, like the reddeningfree $Q$ index (Daflon et al. 1999; Lyubimkov et al. 2002) or small-band Strömgren photometry, as the $T_{\text {eff }}([u-b])$ calibration of Napiwotzki et al. (1993). The required Johnson and Strömgren magnitudes for the individual stars are adopted from the SIMBAD database.

The discrepancies in $T_{\text {eff }}$ can be large, up to $\sim 4000 \mathrm{~K}$, much larger than the offsets studied in Sect. 3.4. The differences in abundance from C II and C III can then easily achieve $\sim 0.7$ dex, similar to what is found in previous studies. Our $T_{\text {eff-values are }}$ typically higher than those from the photometric estimates. The present sample is too small to facilitate an improved $T_{\text {eff }}(Q)$ calibration on its own. However, there is an indication that giants have cooler atmospheres than dwarf stars at the same photometric index. This qualitatively agrees with the findings of Lyubimkov et al. (2002).

Spectroscopic $T_{\text {eff }}$-determinations by Kilian (1992) are also shown in Fig. 15. The approach is conceptually the most similar to the present study despite considerable progress was made over the past 15 years and, as a consequence, gives the closest agreement with our values (except for the cool giant).

These comparisons show that the effective temperatures derived from available photometric calibrations in early B-type stars should be considered only as estimates. If adopted as final temperatures, one should be aware of the potential systematic effects on the abundance determination (see Sect. 3.4). Instead, these could be used as starting points for further refinements via well-understood spectroscopic indicators, in order to bring the model into agreement with all available indicators simultaneously: e.g. multiple $\mathrm{H}$ and $\mathrm{He}$ lines and metal ionization equilibria, as in the present approach. All the atmospheric parameters could then be tightly constrained. Such a step is essential if the $1 \sigma$-uncertainties in the abundance analysis are required to be smaller than $\sim 0.3$ dex.

\section{The stellar present-day $C$ abundance in the solar neighbourhood}

Early-type stars can act as tracers for the present-day chemical composition in the solar neighbourhood. The present small sample of stars is suited to address this topic, as the objects are randomly distributed over nearby $\mathrm{OB}$ associations (three stars in Ori OB1 and Sco-Cen, see Table 2) and the field (the other stars). They are located at distances shorter than $\sim 1 \mathrm{kpc}$ from

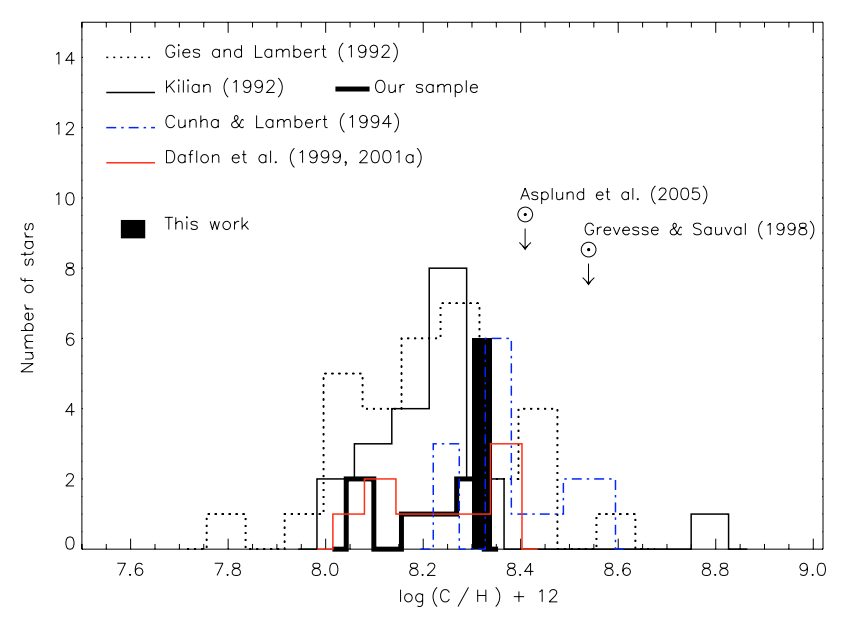

Fig. 16. Comparison of $\mathrm{C}$ abundances derived from our sample of early B-type stars in the solar vicinity (from the field and from OB associations) with results from the literature and the most recent solar values. Similar objects at distances shorter than $\sim 1 \mathrm{kpc}$ from the Sun and at galactocentric distances within up to $\sim 500 \mathrm{pc}$ difference with respect to the location of the Sun are considered. The binsize of the histograms is related to the statistical uncertainty of each sample. Our sample is not large enough for a statistical comparison, therefore a column with a thickness of our uncertainty is displayed. The programme stars coincide with six objects from Kilian (1992): her abundances show a much wider spread for these. See the text for details.

the Sun and at galactocentric distances within up to $\sim 500 \mathrm{pc}$ difference with respect to the location of the Sun. We find a highly-homogeneous carbon abundance of $\varepsilon(C)=8.32 \pm 0.04$ ( $1 \sigma$ statistical uncertainty). Continuing the discussion from the last section, the carbon abundance derived in this work is compared with results from selected previous studies of early B-type dwarf and giant stars in non-LTE, as shown in Fig. 16. These non-LTE analyses, all based on the EB88 model atom but using different linelists, find systematically higher abundances than previous LTE studies (e.g. Kane et al. 1980). However, a wide spread in abundance is still present in each sample. Our results can be directly compared with those of the purely spectroscopic study of Kilian (1992). A mean $\varepsilon(\mathrm{C})=8.19 \pm 0.12$ is derived from the same six stars when adopting her results. This comparison is very important as it shows that a self-consistent analysis, like the one presented here, may drastically reduce the statistical scatter and may also imply a considerable systematic shift of the mean abundance. Similar to Kilian, Gummersbach et al. (1998, not shown in Fig. 16) also performed a consistent spectroscopic analysis of five stars in the solar vicinity as part of their Galactic abundance gradient study. Their results also indicate systematically lower abundances and a considerable spread.

Examples of non-LTE analyses for larger samples of stars based on effective temperatures derived from photometric calibrations are also displayed in Fig. 16. Gies \& Lambert (1992) and Cunha \& Lambert (1994) derived $T_{\text {eff }}$ from calibrations based on Strömgren photometry and non-LTE abundances from the $\mathrm{C}$ II multiplet around $\lambda 5145 \AA$ and where possible from C II $\lambda \lambda 5648 / 62 \AA$. Both multiplets originate in the quartet spin system and they are almost unaffected by non-LTE effects. For the comparison we excluded the five supergiant stars of the sample of Gies \& Lambert (1992). Gies \& Lambert (1992) have two stars in common with the present sample, indicating systematically lower abundances: for HR $2928 \Delta \varepsilon(\mathrm{C}) \simeq-0.3 \mathrm{dex}$ and for HR $1861 \Delta \varepsilon(C) \simeq-0.2$ dex (from the same lines). They 
also consider the doublet C II $\lambda \lambda 6578 / 82 \AA$ for several stars of their sample, but obtain even lower abundances compared to the lines from the quartet spin system (by approximately another $-0.3 \mathrm{dex}$ ). Cunha \& Lambert (1994) have only one star in common with us, HR 1861. They found a similar abundance for this star as Gies \& Lambert (1992). More recently, Daflon et al. (1999, 2001a) derived temperatures from their calibration of the Johnson $Q$-parameter and non-LTE abundances from the C II multiplet around $\lambda 5145 \AA$.

All the data on carbon abundances from early-type stars summarised above was derived from interpretation of observation, which may be affected by many sources of systematic error (see Sect. 3). These data are interpreted in turn to test models of massive star evolution and the chemical evolution of the Galaxy, which are anchor points for studies of stellar and galactochemical evolution in general. Any misinterpretation in the first step in this chain may have severe consequences for our understanding of the whole picture. In this context, it is important to investigate the conclusions drawn from a diagram like Fig. 16.

Previous non-LTE studies all show a broad range of carbon abundances in the solar neighbourhood, spanning a factor of $\sim 10$ in total. Current models of stellar evolution and chemical evolution of the Galaxy do not provide a physical explanation to the large range shown in Fig. 16 for this kind of star located in the solar vicinity ${ }^{7}$. If this extended distribution were real, its physical interpretation would require accounting for the following physical processes. I) Mechanisms that alter the atmospheric structure, such as sufficiently strong magnetic fields or diffusion/radiative levitation processes acting on short timescales and independent of atmospheric parameters and evolutionary age. These have so far been discussed only for a few cases, wellmotivated by observational evidence. A common occurrence of these processes would imply that the basic assumption of homogeneity of the atmosphere may be invalid, and as a consequence the modelling techniques applied so far may be inadequate. II) Extremely efficient depletion mechanisms in the course of stellar evolution, by one to two orders of magnitude larger than currently predicted (e.g. Meynet \& Maeder 2003). Already on the main sequence, mixing with matter from the stellar core would require a higher efficiency than currently predicted for convection during the first dredge-up. Eventually, enrichment mechanisms may also be required that contradict nucleosynthesis (the $3 \alpha$-process is not active in OB dwarfs and giants, carbon is instead depleted by the CNO cycle). III) An enormous chemical inhomogeneity of the present-day interstellar material in the solar neighbourhood out of which the stars have been formed. This would require the possibility of changing abundances practically instantly, even within single clusters by amounts that are otherwise attributed to the past $\sim 12$ Gyrs of Galactochemical evolution (e.g. Chiappini et al. 2003).

Concerning the chemical inhomogeneity, non-uniform abundance distributions as a result of galactochemical evolution are also discussed in literature; e.g., Oey (2003) indicates a scatter of 0.13 dex in oxygen abundance for a population at solar metallicity, which is in reasonable agreement with previous observational constraints. However, this is an upper limit, as homogenization processes were neglected entirely, such that the true scatter can be expected to be much lower.

The present work, in combination with Papers I and II, avoids all these fundamental problems. It implies homogeneous abundances after improving the modelling and the analysis

\footnotetext{
7 When considering also LTE results (e.g. Kane et al. 1980) the whole abundance range rises up to $1.6 \mathrm{dex}$ (a factor of 40).
}

Table 4. Carbon abundances of different objects in the solar vicinity.

\begin{tabular}{lcl}
\hline \hline Objects & $\varepsilon(\mathrm{C})$ & Source \\
\hline B stars (pristine value) & $8.35 \pm 0.05$ & present work \\
B stars & $8.25 \pm 0.08$ & Herrero (2003) \\
Orion H II (gas) & $8.42 \pm 0.02$ & Esteban et al. (2004) \\
Orion H II (gas+dust) & $8.52 \pm 0.02$ & Esteban et al. (2004) \\
young F and G stars & $8.55 \pm 0.10$ & Sofia \& Meyer (2001) \\
ISM & $8.15 \pm 0.06$ & Sofia \& Meyer (2001) \\
Sun & $8.39 \pm 0.05$ & Asplund et al. (2005) \\
Sun & $8.52 \pm 0.06$ & Grevesse \& Sauval (1998) \\
\hline
\end{tabular}

methodology, bringing all model aspects into agreement with observation at once, which has not been achieved so far.

I) It conforms to the finding of a uniform gas-phase carbon abundance in the ISM (out to distances of $1.5 \mathrm{kpc}$, e.g. Sofia \& Meyer 2001, and references therein), with the systematic offset between the two values being a consequence of dust formation in the ISM. The uncertainties of the ISM abundance (see Table 4) are determined by the accuracy to which the oscillator strengths of the resonance lines in the UV are known, and should not exceed more than $\sim 10 \%$.

II) It also agrees with Galactochemical evolution models, which predict homogeneous abundances in the solar neighbourhood (e.g. Chiappini et al. 2003). A variety of hydrodynamic processes should keep the ISM chemically well-mixed on small time scales (Edmunds 1975; Roy \& Kunth 1995). The variation due to the Galactic abundance gradient should amount to up to $\sim 0.04-0.08 \mathrm{dex} \mathrm{kpc}^{-1}$, which is similar to the uncertainty found here (one kpc is the maximum Galactocentric distance sampled in our comparison).

III) Another aspect concerns the notably sub-solar abundances from early-type stars found so far (e.g. Herrero 2003, with respect to the old solar standard, Grevesse \& Sauval 1998 - see Table 4). The present findings remedy the situation, in particular if accounting for the recently revised solar carbon abundance (Asplund et al. 2005, see also Table 4).

IV) Finally, our statistical uncertainty for the whole sample of stars is consistent with the expected variations in surface carbon abundance from stellar evolution considerations. Rotationally-induced mixing with $\mathrm{CN}$-processed material from the core may change the atmospheric composition on the surface. Theory predicts a depletion of carbon by $\sim 0.03$ dex for a star of $20 M_{\odot}$ with initial rotational velocity of $300 \mathrm{~km} \mathrm{~s}^{-1}$ evolving from the zero-age Main Sequence to the end of the Main Sequence stage, reaching $\sim 0.15 \mathrm{dex}$ in the supergiant stage (e.g. Meynet \& Maeder 2003). The presence of a magnetic field may amplify rotational mixing (Maeder \& Meynet 2005); however, the effects on abundances are not expected to exceed a factor $\sim 2$. As absolute rotational velocities of stars can be measured only for a few exceptionally cases, this topic can be addressed comprehensively only by a statistical approach. Our sample of stars is not large enough for this. We note however, that we do not find a significant trend in carbon abundances with evolutionary age for these apparently slow-rotating stars (only $\tau$ Sco is suggested to be a real slow rotator, Donati et al. 2006). The abundances derived may not correspond to the pristine values nonetheless, but it is unlikely that all the stars are very fast rotators seen poleon. Small corrections by up to $\sim+0.05$ dex per star (depending on evolution stage) are predicted by theory for objects of average rotation. We estimate a correction of +0.03 dex to the average value of our sample (considering a small increase in the 
uncertainty), in order to derive an average pristine abundance of $\varepsilon(C)=8.35 \pm 0.05$.

The average pristine carbon abundance from early B-type stars in the solar neighbourhood is compared with other indicators in Table 4. The value is not only in agreement with the revised solar abundance but also with the gas-phase abundance derived for the Orion nebula. The finding of a uniform abundance agrees with a homogeneous ISM abundance. A disagreement is found only with abundances from young F \& G-type stars. This may be resolved when hydrodynamic 3D-analyses of late-type stars become routine, as the discrepancy is similar to the difference between the old and the revised solar standard, which is the result of such an improved analysis. The conclusion of Sofia \& Meyer (2001) that B-type stars are not reliable proxies for present-day abundances in the solar neighbourhood may need a revision in view of the present results.

Despite the small sample size analysed so far, our accurate results indicate that the sub-solar average value and the large scatter of $\mathrm{C}$ abundances in early-type stars found in previous non-LTE studies could be mostly a consequence of systematic uncertainties. These may be easily introduced by the choice of inappropriate atomic data and/or stellar parameters, as we have shown. However, a confirmation of our findings from analysis of a larger sample of stars is required, also for other elements.

\section{Summary and conclusions}

The motivation of this work was to solve a long-standing problem in stellar astrophysics: the reliable determination of carbon abundances from early-type stars. For this purpose we constructed a sophisticated C II/III/IV model atom for non-LTE line-formation calculations based on input atomic data that were carefully selected by a calibration process. This was performed through an extensive iteration scheme that allowed us to constrain not only the input atomic data but also simultaneously the atmospheric parameters of our calibration stars, the basis for all further studies. The calibration sample consists of six bright and apparently slow-rotating early B-type stars in the solar neighbourhood, with high-S/N, high-resolution spectra and a broad wavelength coverage.

The self-consistent analysis provides atmospheric parameters with unprecedented accuracy and with reduced systematic error: for effective temperature, the uncertainties are as low as $\sim 1 \%$ and for surface gravity $\sim 10 \%$. The atmospheric parameters derived from the carbon ionization equilibria are highly consistent with our previous spectroscopic analyses: results from a non-LTE study of $\mathrm{H}$ and $\mathrm{He}$ in the visual and in the near-IR, including establishing the He I/II ionization equilibrium in the hotter stars (Paper I) and reproducing the spectral energy distributions from the UV to the near-IR (Paper II).

All C lines considered, from up to three ionization stages, indicate similar abundances. The linelist includes 40 transitions suitable for analysis over a wide wavelength range. In particular, the strongest features, of highest importance for extragalactic applications, are consistently modelled. The statistical $1 \sigma$-uncertainties of the $\mathrm{C}$ abundance in each star are of the order $\sim 0.05-0.10$ dex. We estimate the systematic $1 \sigma$ uncertainties to be $\sim 0.10-0.15 \mathrm{dex}$. The average carbon abundance from $\mathrm{CII} / \mathrm{III} / \mathrm{IV}$ in the sample stars is highly uniform, $\varepsilon(\mathrm{C})=8.32 \pm 0.04$.

These results of unprecedented precision provide important constraints for stellar evolution and the chemical evolution of the Galaxy, despite the small sample size. I) They suggest that carbon depletion due to rotational mixing in the course of stellar evolution is small for stars without excessive initial angular momentum (by $<0.05$ dex) up to the giant stage, in agreement with theoretical predictions. II) In consequence they suggest that the present-day carbon abundance in the solar neighbourhood is higher and much more homogeneous than indicated by previous work on early-type stars. This is consistent with the uniform abundance found in studies of the ISM and with predictions from models of the chemical evolution of the Galaxy. Moreover, our re-evaluated stellar value agrees with the gas-phase abundance derived for the Orion H II region and the recently revised solar abundance.

This work shows that one should not underestimate the importance of a careful choice of input atomic data for non-LTE analyses. Accurate data are required not only for radiative transitions but also for collisional transitions. The carbon abundance analysis also turned out to be highly sensitive to the choice of atmospheric parameters, in particular to effective temperatures. If not accounted for properly, both factors may result in systematic errors in the interpretation of observed spectra, which cannot be reduced by statistics, i.e. an increase in the sample size of stars to be analysed. Detailed comparisons and calibrations of models with observed spectra are highly important, as these constitute the only empirical constraints for quantitative analyses. Only then can stellar and Galactochemical evolution models be verified in a meaningful way, and the studies be extended to stars in other galaxies.

Acknowledgements. The authors wish to thank U. Heber and K. Cunha for their interest in and their support of the project. We further thank K. Butler for making available DETAIL and Surface and M. Altmann for kindly providing the FEROS data. M.F.N. thanks S. Daflon for encouraging the study of this subject and also acknowledges a Ph.D. scholarship by the Deutscher Akademischer Austausch Dienst (DAAD).

\section{References}

Aggarwal, K. M., \& Keenan, F. P. 2004, Phys. Scr., 69, 385

Allen, C. W. 1973, Astrophysical Quantities, 3rd edn. (London, Athlone Press) Anders, E., \& Grevesse, N. 1989, Geochim. Cosmochim. Acta, 53, 197

Andrievsky, S. M., Korotin, S. A., Luck, R. E., \& Kostynchuk, L. Yu. 1999, A\&A, 350, 598

Asplund, M., Grevesse, N., \& Sauval, A. J. 2005, in Cosmic Abundances as Records of Stellar Evolution and Nucleosynthesis, ed. T. G. Barnes III, \& F. N. Bash (San Francisco: ASP), 25

Barnett, E. W., \& McKeith, C. D. 1988, MNRAS, 234, 325

Becker, S., \& Butler, K. 1990, A\&A, 235, 326

Burbidge, E. M., Burbidge, G. R., Fowler, W. A., \& Hoyle, F. 1957, Rev. Modern Phys., 29, 547

Butler, K., \& Giddings, J. R. 1985, in Newsletter of Analysis of Astronomical Spectra, No. 9, Univ. London

Cameron, A. G. 1957, PASP, 69, 201

Chiappini, C., Matteucci, F., \& Romano, D. 2001, ApJ, 554, 1044

Chiappini, C., Romano, D., \& Matteucci, F. 2003, MNRAS, 339, 63

Cowley, C. 1971, Observatory, 91, 139

Cunha, K., \& Lambert, D. L. 1994, ApJ, 426, 170

Daflon, S., \& Cunha, K. 2004 ApJ, 617, 1115

Daflon, S., Cunha, K., \& Becker, S. R. 1999, ApJ, 522, 950

Daflon, S., Cunha, K., Becker, S. R., \& Smith, V. V. 2001a, ApJ, 552, 309

Daflon, S., Cunha, K., Butler, K., \& Smith, V. V. 2001b, ApJ, 563, 325

Donati, J. F., Howarth, I. D., Jardine, M. M., et al. 2006, MNRAS, 370, 629

Eber, F. 1987, Ph.D. Thesis, Munich Observatory, University of Munich

Eber, F., \& Butler, K. 1988, A\&A, 202, 153 (EB88)

Edmunds, M. G. 1975, Ap\&SS, 32, 483

Esteban, C., Peimbert, M., García-Rojas, J., et al. 2004, MNRAS, 355, 229

Froese Fischer, C., \& Tachiev, G. 2004, At. Data Nucl. Data Tables, 87, 1 (FFT04)

Gies, D. R., \& Lambert, D. L. 1992, ApJ, 387, 673

Giddings, J. R. 1981, Ph.D. Thesis, University of London

Grevesse, N., \& Sauval, A. J. 1998, Space Sci. Rev., 85, 161

Griem, H. R. 1974, Spectral line broadening by plasmas (New York and London, Academic Press). 
Grigsby, J. A., Morrison, N. D., \& Anderson, L. S. 1992, ApJS, 78, 205

Gray, D. F. 1992, Observations and Analysis of Stellar Photospheres, 2nd edition (Cambridge: Cambridge University Press)

Gummersbach, C. A., Kaufer, A., Schäfer, D. R., Szeifert, T., \& Wolf, B. 1998 , A\&A, 338, 881

Hardorp, J., \& Scholz, M. 1970, ApJS, 173, 19

Heger, A., \& Langer, N. 2000, ApJ, 544, 1016

Herrero, A. 2003, in CNO in the Universe, ed. C. Charbonnel, D. Schaerer, \&

G. Meynet (San Francisco: ASP), 10

Hou, J. J., Prantzos, N., \& Boissier, S. 2000, A\&A, 362, 921

Hunter, I., Dufton, P. L., Smartt, S. J., et al. 2007, A\&A, 466, 277

Kane, L., McKeith, C. D., \& Dufton, P. L. 1980, A\&A, 84, 115

Kilian, J. 1992, A\&A, 262, 171

Kodaira, K., \& Scholz, M., 1970, A\&A, 6, 93

Korn, A. J., Keller, S. C., Kaufer, A., et al. 2002, A\&A, 385, 143

Korn, A. J., Nieva, M. F., Daflon, S., \& Cunha, K. 2005, ApJ, 633, 899

Kurucz, R. L. 1993a, CD-ROM No. 2-12 (Cambridge, Mass.: SAO)

Kurucz, R. L. 1993b, CD-ROM No. 13 (Cambridge, Mass.: SAO)

Kurucz, R. L., \& Bell, B. 1995, CD-ROM No. 23 (Cambridge, Mass.: SAO)

Lambert, D. L. 1993, Phys. Scr., T47, 186

Lanz, T., \& Hubeny, I. 2003, ApJS, 146, 417

Lanz, T., \& Hubeny, I. 2007, ApJS, 169, 83

Lennon, D. L. 1983, MNRAS, 205, 829

Lyubimkov, L. S., Rachkovskaya, T. M., Rostopchin, S. I., \& Lambert, D. L. 2002, MNRAS, 333, 9 (LRRL)

Maeder, A., \& Meynet, G. 2000, ARA\&A, 38, 143

Maeder, A., \& Meynet, G. 2005, A\&A, 440, 1041

Meynet, G., \& Maeder, A. 2003, A\&A, 404, 975

Mihalas, D. 1978, Stellar Atmospheres, 2nd edition (San Francisco: Freeman)

Mitnik, D. M., Griffin, D. C., Ballance, C. P., \& Badnell, N. R. 2003, J. Phys. B 36, 717

Moore, C. E. 1993, in CRC Handbook of Chemistry and Physics, 76th ed. J. W. Gallagher (Boca Raton: CRC)

Nahar, S. 2002a, At. Data Nucl. Data Tables, 80, 205 (N02a)

Nahar, S. 2002b, A\&A, 389, 716 (N02b)

Nahar, S., \& Pradhan, A. 1997, ApJS, 111, 339 (NP97)

Napiwotzki, R., Schönberner, D., \& Wenske, V. 1993, A\&A, 268, 653
Nieva, M. F., \& Przybilla, N. 2006, ApJ, 639, L39 (Paper II)

Nieva, M. F., \& Przybilla, N. 2007, A\&A, 467, 295, (Paper I)

Oey, M. S. 2003, in A Massive Star Odyssey: From Main Sequence to Supernova, ed. K. van der Hucht, A. Herrero, \& C. Esteban (San Francisco: ASP), 620

Przybilla, N. 2005, A\&A, 443, 293

Przybilla, N., \& Butler, K. 2004, ApJ, 609, 1181

Przybilla, N., Butler, K., \& Kudritzki, R. P. 2001, A\&A, 379, 936

Przybilla, N., Butler, K., Heber, U., \& Jeffery, C. S. 2005, A\&A, 443, L25

Przybilla, N., Butler, K., Becker, S. R., \& Kudritzki, R. P. 2006a, A\&A, 445, 1099

Przybilla, N., Nieva, M. F., \& Edelmann, H. 2006b, Balt. Astron., 15, 107

Przybilla, N., Nieva, M. F., Heber, U., \& Jeffery, C. S. 2006c, Balt. Astr., 15, 163 Quinet, P. 1998, A\&AS, 129, 603

Rolleston, W. R., Smartt, S. J., Dufton, P. L., \& Ryans, R. S. 2000, A\&A, 363, 537

Rolleston, W. R., Venn, K., Tolstoy, E., \& Dufton, P. L. 2003, A\&A, 400, 21

Roy, J. R., \& Kunth, D. 1995, A\&A, 294, 432

Ryans, R. S. I., Dufton, P. L., Rolleston, W. R. J., et al. 2002, MNRAS, 336, 577

Rybicki, G. B., \& Hummer, D. G. 1991, A\&A, 245, 171

Sakhibullin, N. A. 1987, Sov. Astron., 31(2), 151

Schöning, T. 1993, A\&A, 267, 300

Seaton, M. J. 1962, in Atomic and Molecular Processes (New York: Ac. Press)

Sigut, T. A. A. 1996, ApJ, 473, 452

Sofia, U. J., \& Meyer, D. M. 2001, ApJ, 554, L221

Trundle, C., Dufton, P. L., Lennon, D. J., Smartt, S. J., \& Urbaneja, M. A. 2002, A\&A, 395, 519

Unsöld, A. 1942, ZAp, 21, 229

Urbaneja, M. A., Herrero, A., Bresolin, F., et al. 2005a, ApJ, 622, 862

Urbaneja, M. A., Herrero, A., Kudritzki, R. P., et al. 2005b, ApJ, 635, 311

Van Regemorter, H. 1962, ApJ, 136, 906

Wiese, W. L., Fuhr, J. R., \& Deters, T. M. 1996, J. Phys. \& Chem. Ref. Data, Mon., 7

Wilson, N. J., Bell, K. L., \& Hudson, C. E. 2005, A\&A, 432, 731

Wilson, N. J., Bell, K. L., \& Hudson, C. E. 2007, A\&A, 461, 765

Yan, Y., \& Seaton, M. J. 1987, J. Phys. B, 20, 6409

Yan, Y., Taylor, K. T., \& Seaton, M. J. 1987, J. Phys. B, 20, 6399 
M. F. Nieva and N. Przybilla: Non-LTE line formation for C II-IV in early B-type stars, Online Material $p 1$

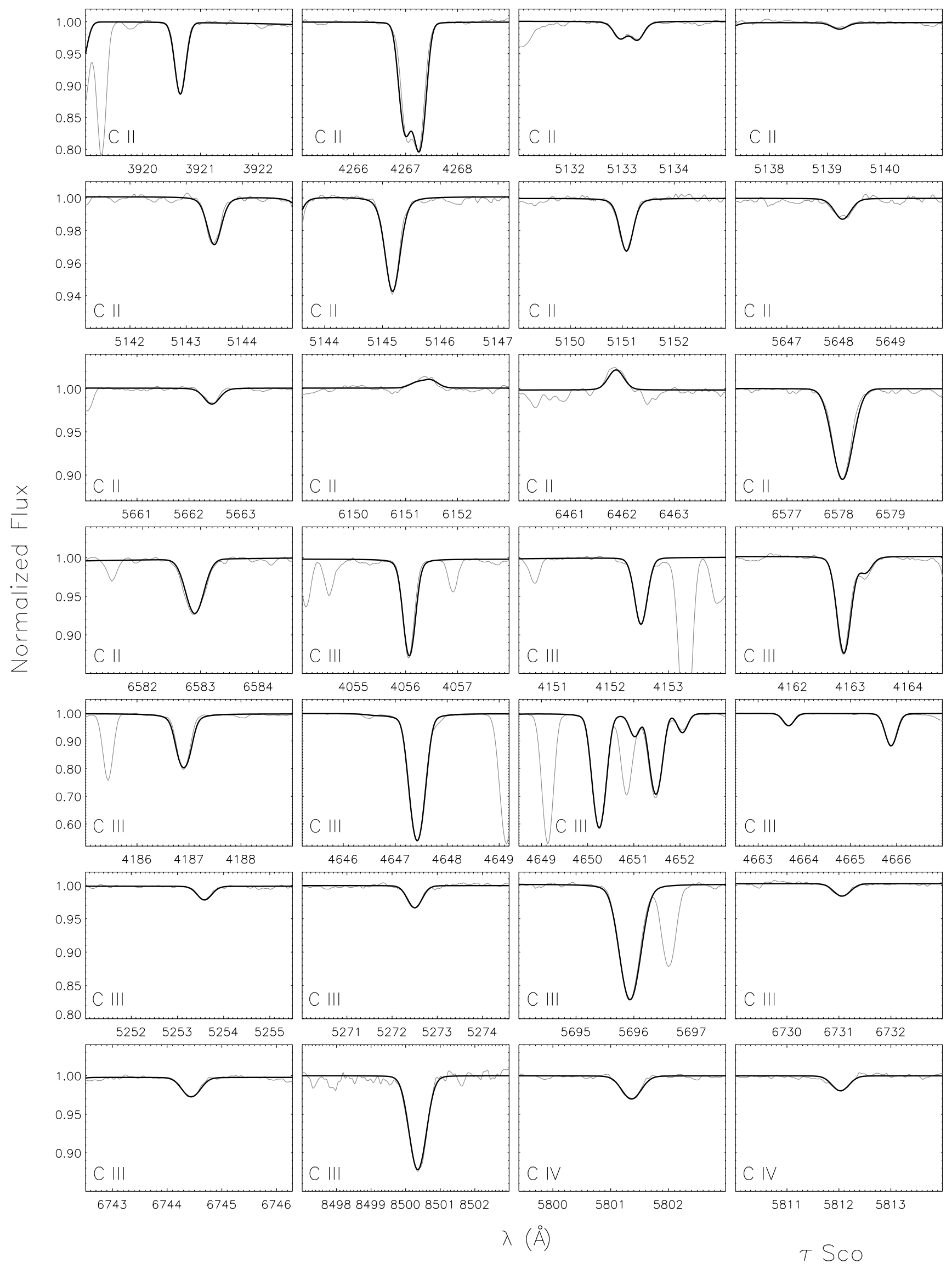

Fig. 17. Examples of our line profile fits (black line) to the observed high-quality spectrum (grey line) of $\tau$ Sco. 
M. F. Nieva and N. Przybilla: Non-LTE line formation for C II-IV in early B-type stars, Online Material p 2

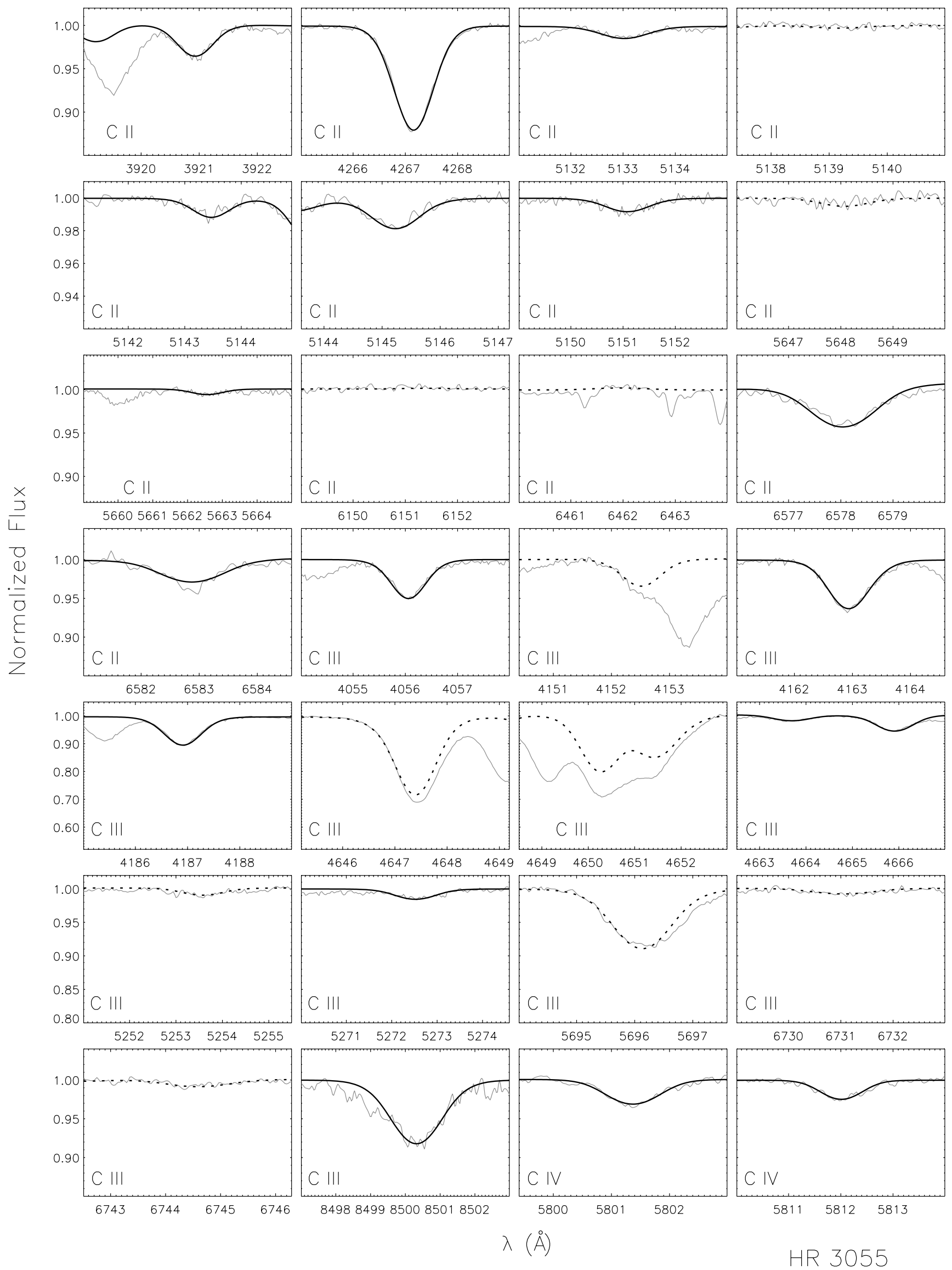

Fig. 18. As Fig. 17, but for HR 3055. Dotted lines indicate the features not included in the abundance determination. 
M. F. Nieva and N. Przybilla: Non-LTE line formation for C II-IV in early B-type stars, Online Material p 3
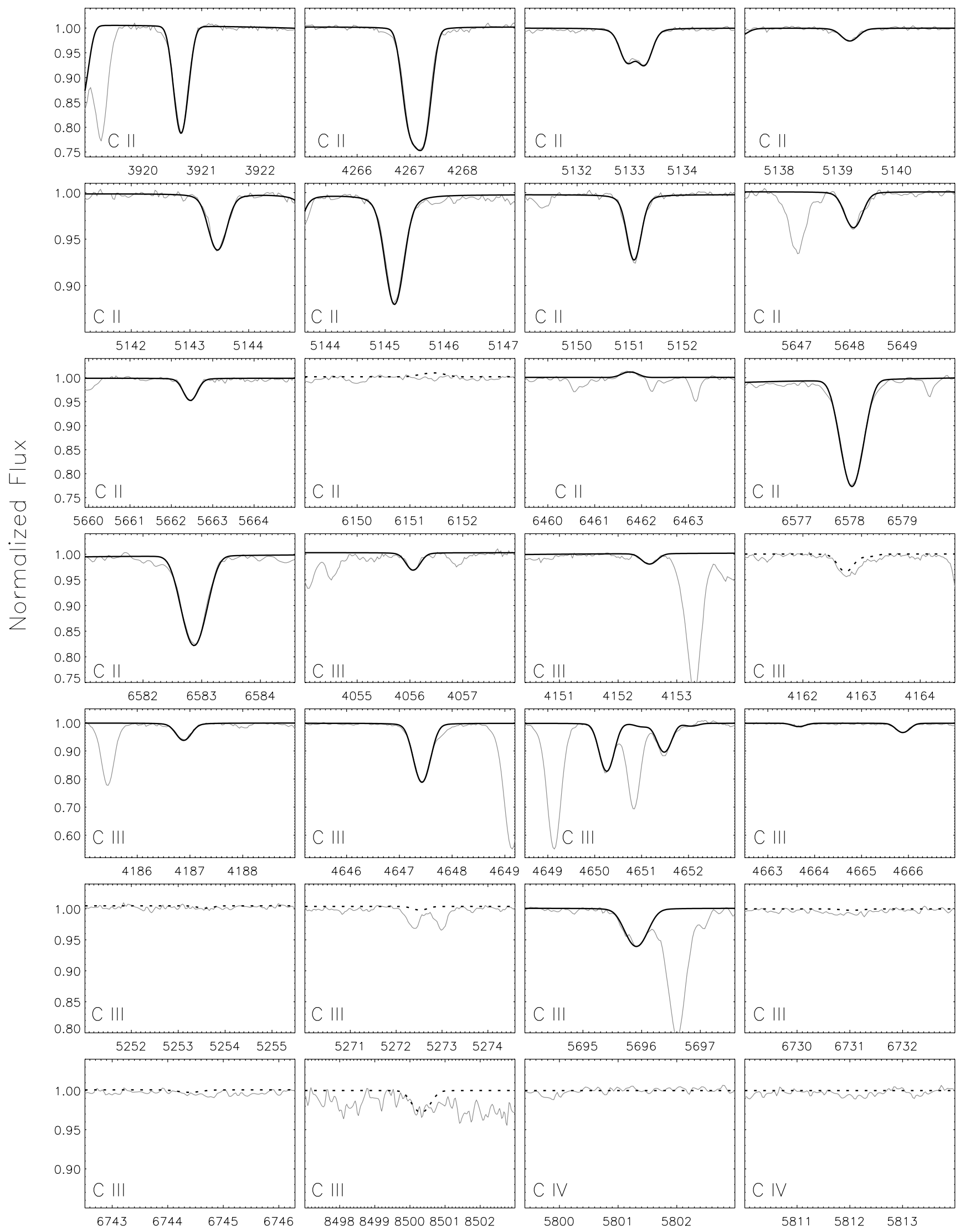

$5138 \quad 5139 \quad 5140$
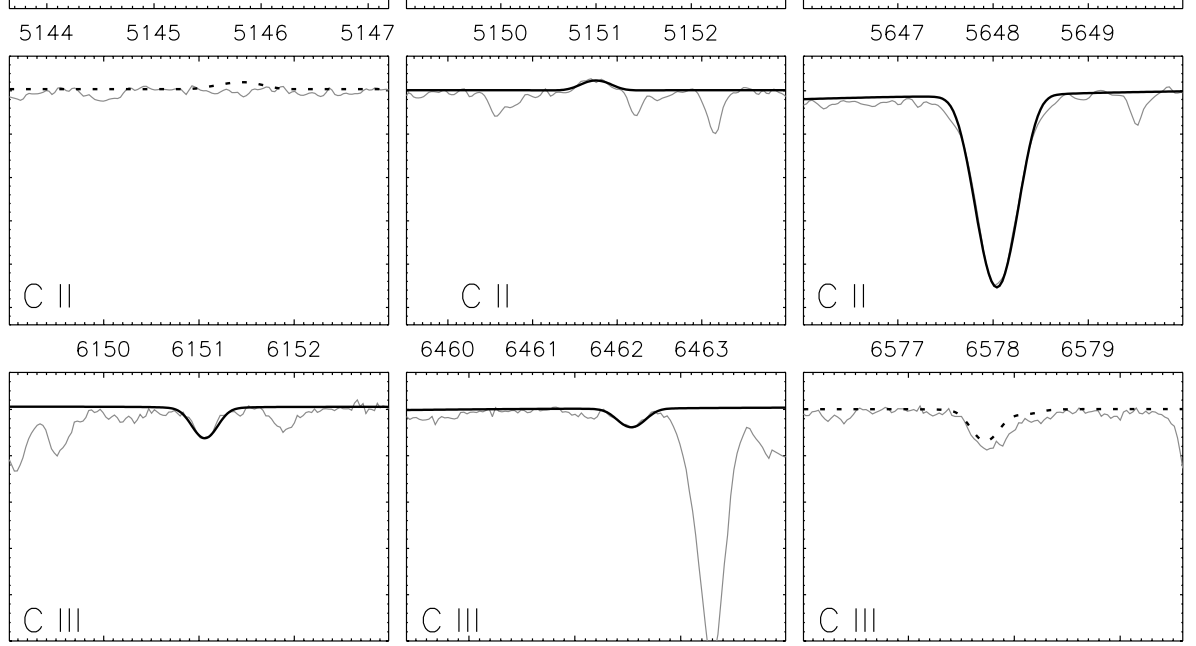

$6460 \quad 6461 \quad 6462 \quad 6463$
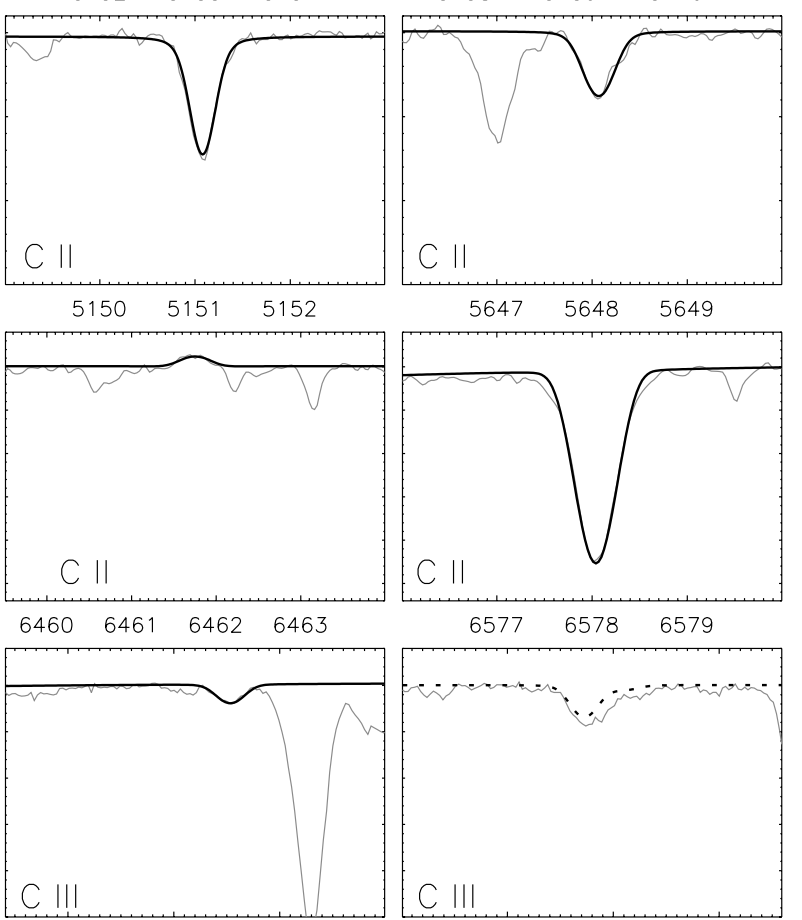

$6577 \quad 6578 \quad 6579$
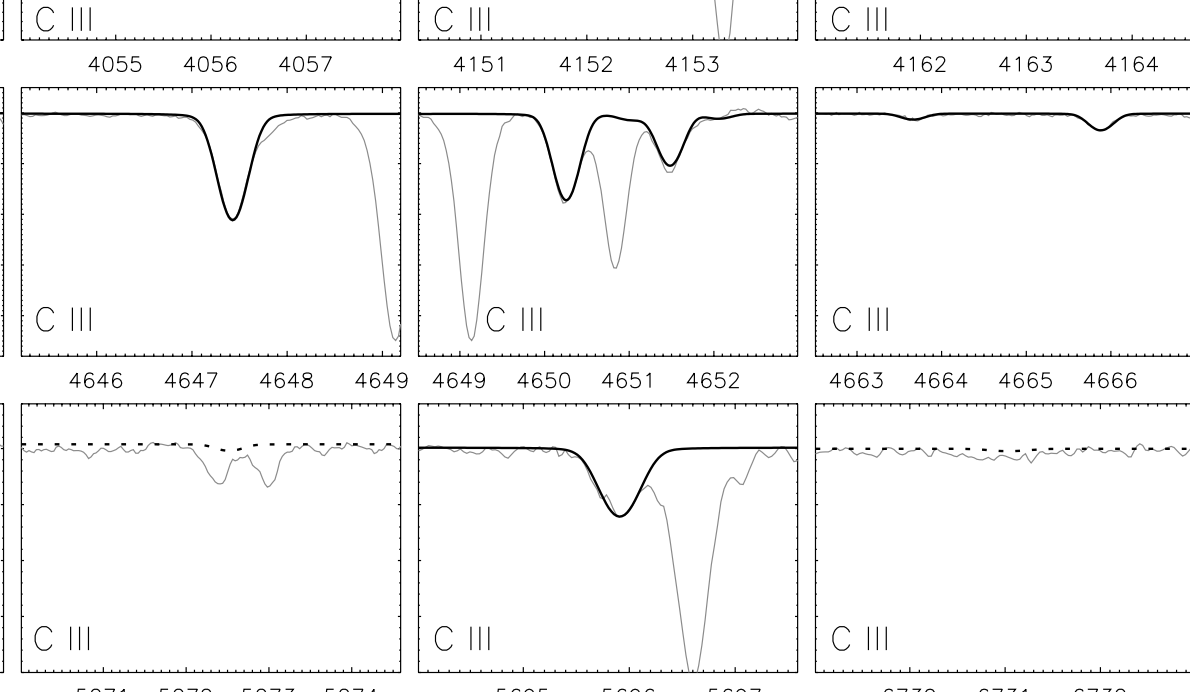

C III

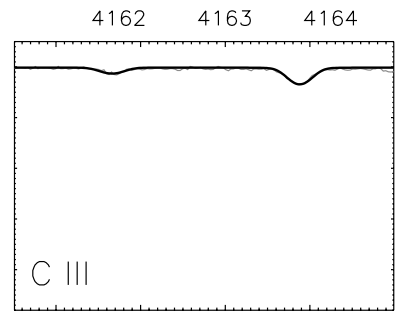

$46634664 \quad 4665 \quad 4666$
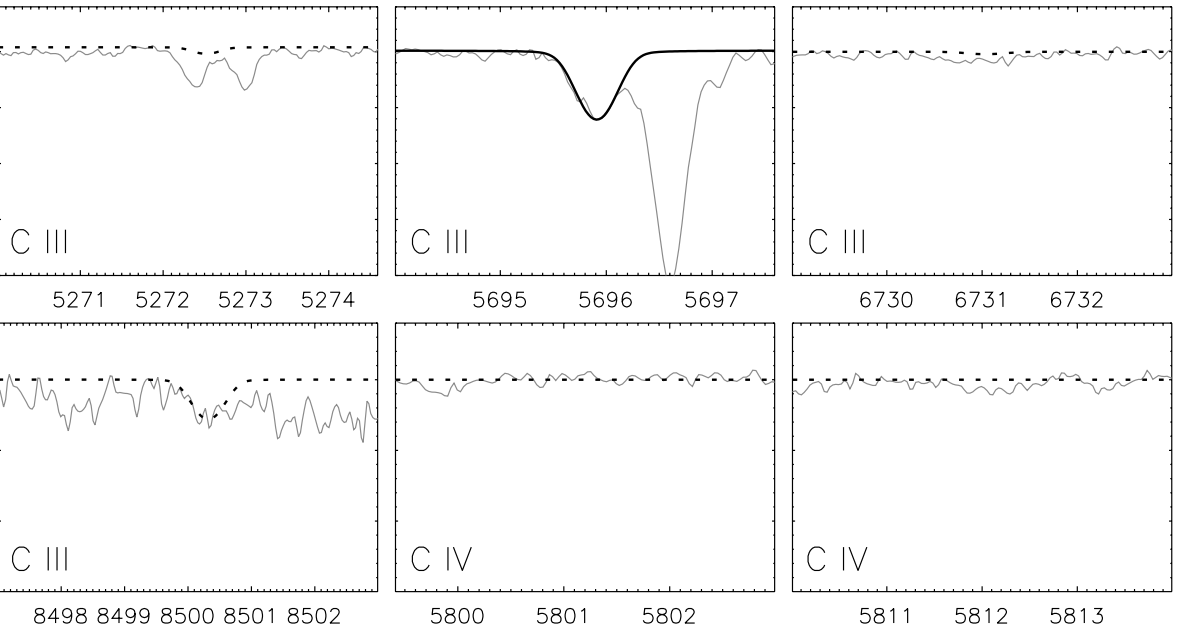

$\lambda(\AA)$

HR 1861

Fig. 19. As Fig. 18, but for HR 1861. 
M. F. Nieva and N. Przybilla: Non-LTE line formation for C II-IV in early B-type stars, Online Material p 4

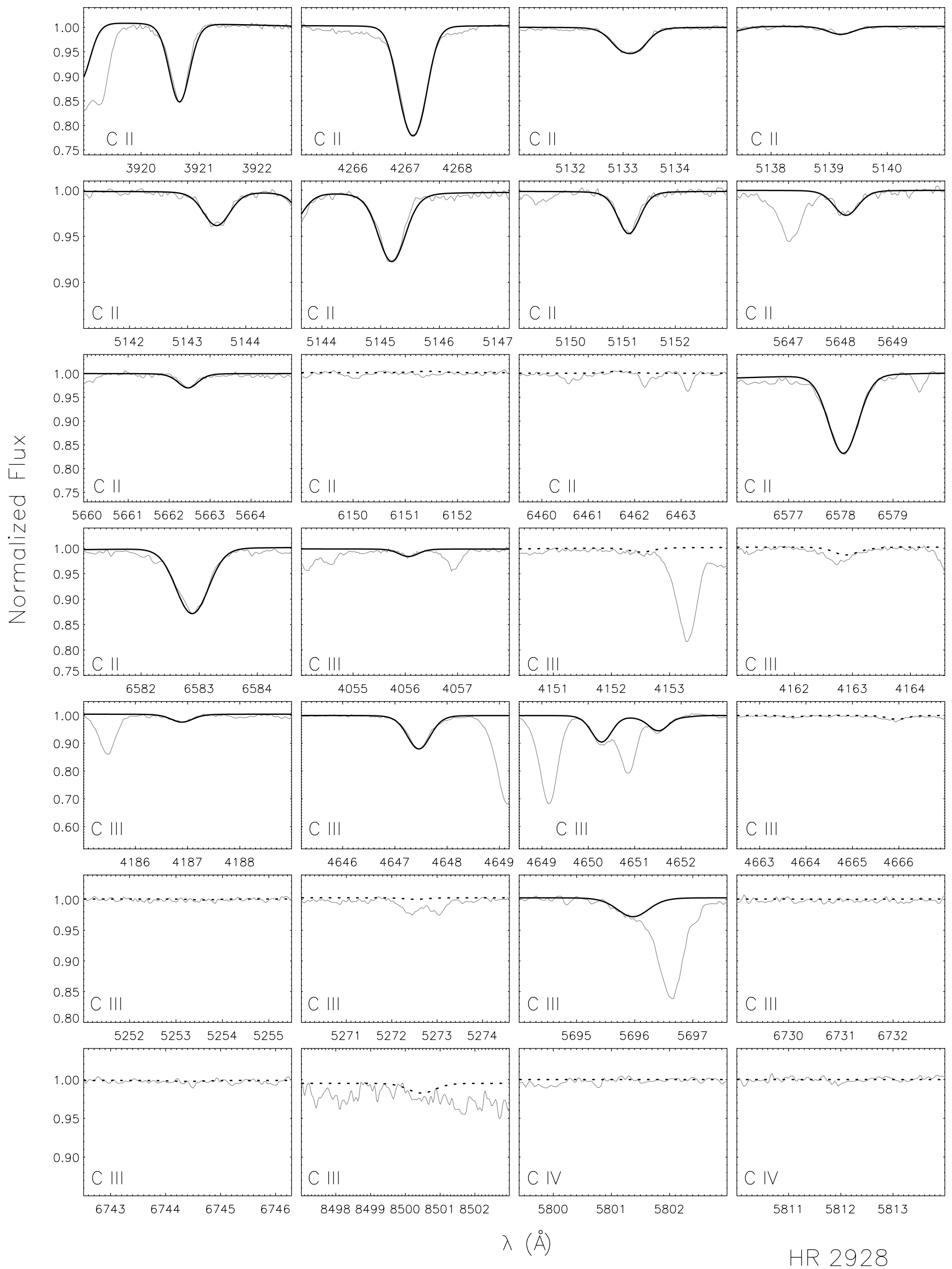

Fig. 20. As Fig. 18, but for HR 2928. 
M. F. Nieva and N. Przybilla: Non-LTE line formation for C II-IV in early B-type stars, Online Material p 5
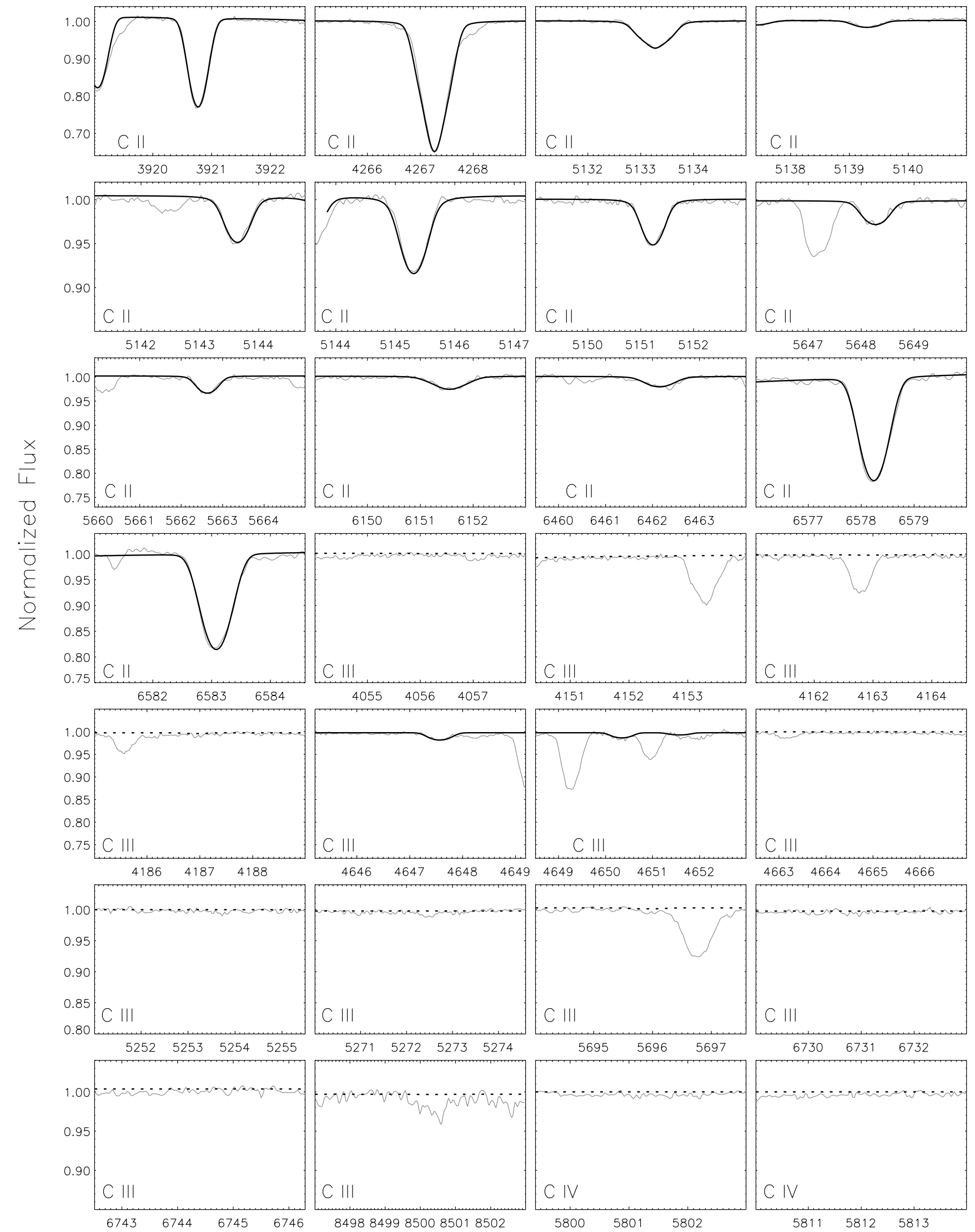

C III

$\lambda(\AA)$

HR 5285

Fig. 21. As Fig. 18, but for HR 5285. 
M. F. Nieva and N. Przybilla: Non-LTE line formation for C II-IV in early B-type stars, Online Material p 6
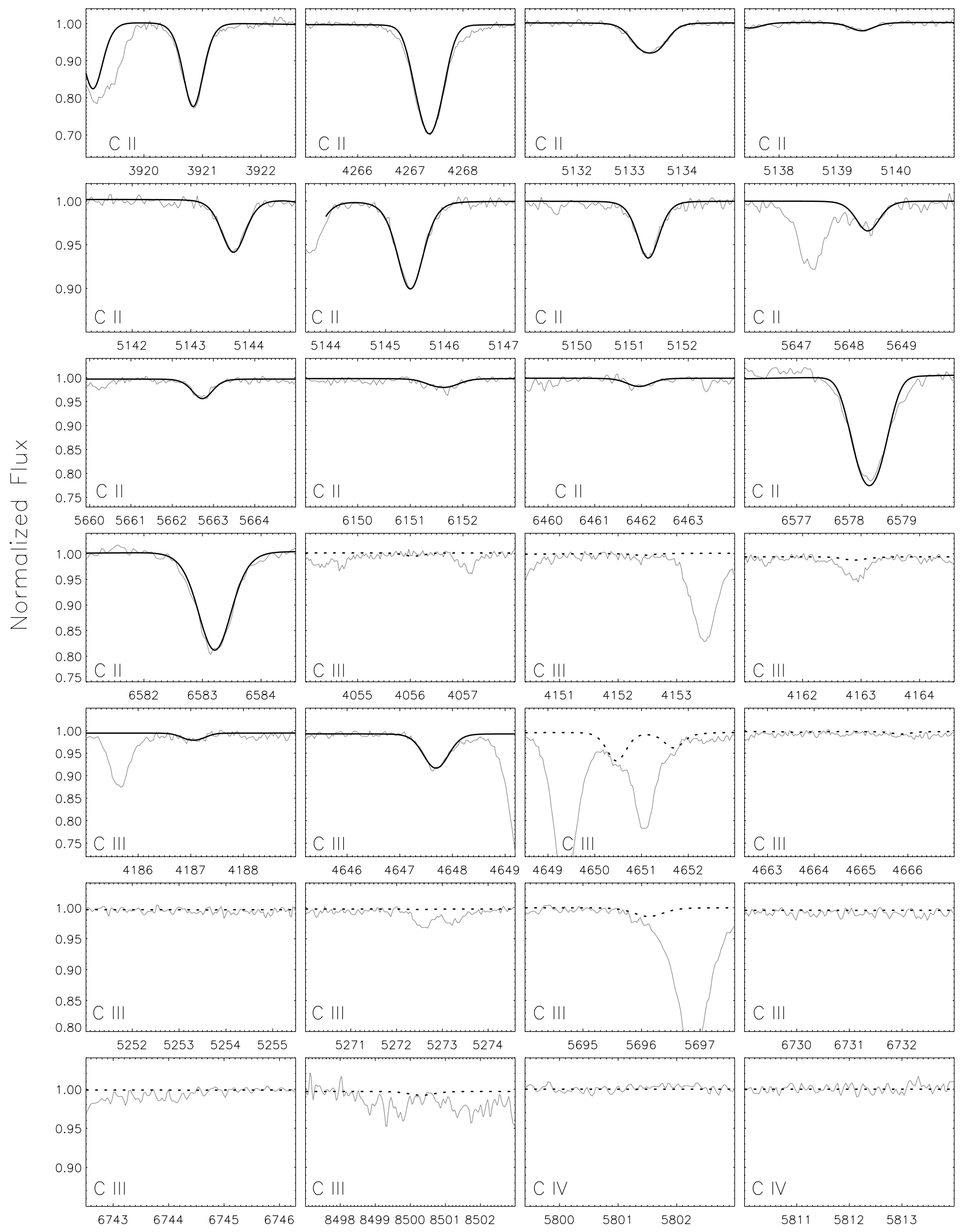

$\lambda(\AA)$

HR 3468

Fig. 22. As Fig. 18, but for HR 3468. 